\title{
ORLICZ SPACES AND THE LARGE SCALE GEOMETRY OF HEINTZE GROUPS
}

\author{
MATIAS CARRASCO PIAGGIO
}

\begin{abstract}
We consider an Orlicz space based cohomology for metric (measured) spaces with bounded geometry. We prove the quasi-isometry invariance for a general Young function. In the hyperbolic case, we prove that the degree one cohomology can be identified with an Orlicz-Besov function space on the boundary at infinity. We give some applications to the large scale geometry of homogeneous spaces with negative curvature (Heintze groups). As our main result, we prove that if the Heintze group is not of Carnot type, any self quasi-isometry fixes a distinguished point on the boundary and preserves a certain foliation on the complement of that point.
\end{abstract}

Keywords: Orlicz spaces, Heintze groups, quiasi-isometry invariants, $\delta$-hyperbolicity. 2010 SubJeCt Classification: 20F67, 30Lxx, 46E30, 53C30.

\section{INTRODUCTION}

In this article we are interested in the large scale geometry of Heintze groups. Homogeneous manifolds with negative sectional curvature where characterized by Heintze in [Hei74]. Each such manifold is isometric to a solvable Lie group $X_{\alpha}$ with a left invariant metric, and the group $X_{\alpha}$ is a semi-direct product $N \rtimes_{\alpha} \mathbb{R}$ where $N$ is a connected, simply connected, nilpotent Lie group, and $\alpha$ is a derivation of $N$ whose eigenvalues all have positive real parts. Such a group is called a Heintze group.

A purely real Heintze group is a Heintze group $X_{\alpha}$ as above, for which the action of $\alpha$ on the Lie algebra of $N$ has only real eigenvalues. Every Heintze group is quasi-isometric to a purely real Heintze group, unique up to isomorphism, see [Cor12, Section 5B]. In the sequel we will focus only on purely real Heintze groups.

Let $\mathfrak{n}$ be the Lie algebra of $N$, and $\operatorname{Der}(\mathfrak{n})$ be the Lie algebra of derivations on $\mathfrak{n}$. We denote by Exp : $\operatorname{Der}(\mathfrak{n}) \rightarrow \operatorname{Aut}(\mathfrak{n})$ the matrix exponential. The group structure of $X_{\alpha}$ is then given by a contracting action $\tau: \mathbb{R} \rightarrow \operatorname{Aut}(N)$, where $\tau$ satisfies $d_{e} \tau(t)=\operatorname{Exp}(-t \alpha)$.

We will use the notation $(x, t)$ to denote a point of $X_{\alpha}$. Any left invariant metric on $X_{\alpha}$ is Gromov hyperbolic, since any two such metrics are bi-Lipschitz equivalent. Assume that $X_{\alpha}$ is equipped with a left invariant metric for which the vertical lines $t \mapsto(x, t)$ are unitspeed geodesics. They are all asymptotic when $t \rightarrow-\infty$, and hence, they define a "special" boundary point denoted $\infty$. The boundary at infinity $\partial X_{\alpha}$ is a topological $n$-sphere, and can be therefore identified with the one-point compactification $N \cup\{\infty\}$. The left action of $X_{\alpha}$ on its boundary has two orbits, namely, $N$ and $\infty$.

Two general problems motivate this work: first, to understand QIsom $\left(X_{\alpha}\right)$, the group of self quasi-isometries of $X_{\alpha}$; and second, the quasi-isometric classification of Heintze groups. These problems have been approached by many authors and by means of several methods, see for instance [Ham87, Pan89b, FM00, Pan07, Dym10, CT11, DP11, Pen11, Xie12, SX12, Xie14a]. We refer the reader to [Cor12] for a survey on the subject. 
In this article, we focus on the Pointed Sphere Conjecture [Cor12, Conjecture 6. C. 9]. It states that $\infty$ is fixed by the boundary homeomorphism of any self quasi-isometry of $X_{\alpha}$, unless $X_{\alpha}$ is isometric (for some left invariant metric) to a rank one symmetric space.

The conjecture is known to be true in the following cases. Recall that $X_{\alpha}$ is said to be of Carnot type if the Lie algebra spanned by the eigenvectors corresponding to the smallest eigenvalue of $\alpha$ is the whole algebra $\mathfrak{n}$ [Cor12, Definition 2. G. 1].

- Pan89b, Corollary 6.9], the conjecture holds whenever $X_{\alpha}$ is not of Carnot type and $\alpha$ is diagonalizable.

- Xie12, SX12, Xie14a, the conjecture holds when $N \simeq \mathbb{R}^{n}$ is abelian.

- [Xie13, the conjecture holds when $N \simeq H_{2 n+1}$ is the real Heisenberg group of dimension $2 n+1$ and $\alpha$ is diagonalizable. Also in [Xie14b], the conjecture is shown to be true for a non-diagonalizable derivation when $n=1$.

The idea behind the proofs is similar in all the three cases. It consists in finding a quasiisometry invariant foliation on $\partial X_{\alpha}$ which is singular at the point $\infty$. The leaves of this foliation are the accessibility classes of points by rectifiable curves, with respect to an appropriate visual metric on the boundary. To this end, Pansu consider $L^{p}$-cohomology, and Xie consider the $p$-variation of functions on the boundary.

Following an original idea of Romain Tessera, and Pansu's methods, we propose here an approach based on the theory of Orlicz spaces [RR91. This allows us to extend these results to all Heintze groups which are not of Carnot type. We mention that Orlicz spaces based cohomologies have been considered recently also in [Kop13, KP13].

The next theorem is our main result. Let $\mu_{1}$ be the smallest eigenvalue of $\alpha$, and let $H_{1}$ be the closed connected subgroup of $N$ whose Lie algebra is spanned by the $\mu_{1}$-eigenvectors belonging to the $\mu_{1}$-Jordan blocks of maximal size. It is a non-trivial and proper subgroup of $N$ when $X_{\alpha}$ is not of Carnot type, see Section 1.2 for more details.

Theorem 1.1. Let $X_{\alpha}$ be a purely real Heintze group that is not of Carnot type. The boundary homeomorphism of any self quasi-isometry of $X_{\alpha}$ fixes the special boundary point $\infty$. Furthermore, in this case, it preserves the left cosets of the subgroup $H_{1}$.

Another important ingredient in our approach is a localization technique. Let us motivate it by considering the following examples. Let $N=\mathbb{R}^{2}$ and consider the Heintze group $X_{i}:=X_{\alpha_{i}}, i=1,2,3$, where

$$
\text { (i) } \alpha_{1}=\left(\begin{array}{ll}
1 & 0 \\
0 & 1
\end{array}\right) \text { (ii) } \alpha_{2}=\left(\begin{array}{ll}
1 & 0 \\
0 & \mu
\end{array}\right) \text { (iii) } \alpha_{3}=\left(\begin{array}{ll}
1 & 1 \\
0 & 1
\end{array}\right) \text {, }
$$

and $\mu>1$. Notice that $X_{1}$ is isometric to the real hyperbolic space $\mathbb{H}^{3}$. The degree one $L^{p}$-cohomology of $X_{i}$ can be identified with a Besov space on the boundary [Pan89a, BP03]. Let us consider the quasi-isometry invariant Banach algebra of continuous Besov functions $A^{p}\left(\partial X_{i}\right)$. The dependence on $p$ of this algebra is summarized in Figure 1.1 .

One way to isolate the point $\infty$ is suggested by the following construction which appears in [Shc14]. Let $Z_{i}$ be the Heintze cone defined as the quotient space of $X_{i}$ by the discrete group of translations $\mathbb{Z}^{2}$. The boundary $\partial Z_{i}$ is the union of a torus $T_{i}$ and the isolated point $\infty$. The dependence on $p$ of $A^{p}\left(\partial Z_{i}\right)$ is summarized in Figure 1.2 . One would like to define a similar cone with respect to any other point $\xi \in \partial X_{i}$.

The pull-back of a function $u \in A^{p}\left(\partial Z_{i}\right)$ by the projection map is periodic and does not define an element of $A^{p}\left(\partial X_{i}\right)$. Nevertheless, it satisfies a local integrability condition, i.e. it belongs to the Fréchet algebra $A_{\mathrm{loc}}^{p}\left(\partial X_{i} \backslash\{\infty\}\right)$. The key point is that the dependence 


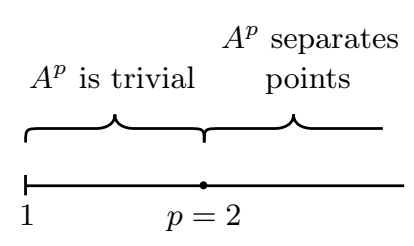

(i)

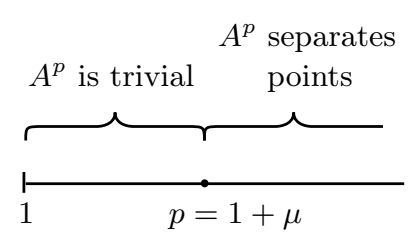

(ii)

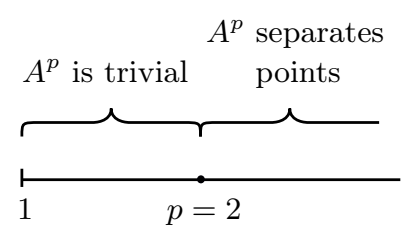

(iii)

Figure 1.1. Dependence on $p$ of $A_{p}\left(\partial X_{i}\right)$.

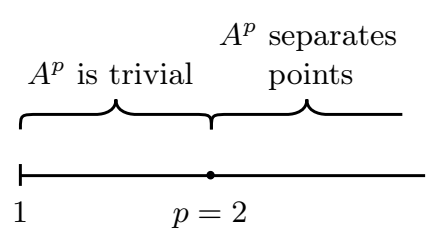

(i) and (iii)

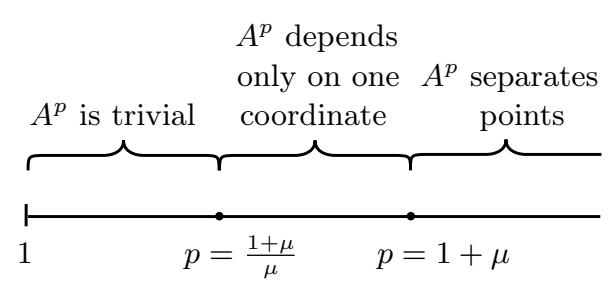

(ii)

Figure 1.2. Dependence on $p$ of $A_{p}\left(\partial Z_{i}\right)$.

on $p$ of the algebras $A_{\text {loc }}^{p}\left(\partial X_{i} \backslash\{\xi\}\right)$ coincides with that summarized in Figure 1.2 when $\xi=\infty$, and with that in Figure 1.1 when $\xi \in \mathbb{R}^{2}$. This explains the case (ii).

Let us look at (iii) in more detail. The parabolic visual metric on $\partial X_{3} \backslash\{\infty\}=\mathbb{R}^{2}$ is bi-Lipschitz equivalent to the function

$$
\varrho\left(\left(x_{1}, y_{1}\right) ;\left(x_{2}, y_{2}\right)\right)=\max \left\{\left|y_{2}-y_{1}\right|,\left|\left(x_{2}-x_{1}\right)-\left(y_{2}-y_{1}\right) \log \right| y_{2}-y_{1}||\right\} .
$$

From this expression, we see that the projection functions, $\pi_{1}(x, y)=x$ and $\pi_{2}(x, y)=y$, have different regularity properties with respect $\varrho$. That is, $\pi_{2}$ is Lipschitz, while $\pi_{1}$ satisfies the inequality

$$
\left|\pi_{1}(v)-\pi_{1}(w)\right| \lesssim \varrho(v, w) \log \left(\frac{1}{\varrho(v, w)}\right) .
$$

In particular, $\pi_{1}$ is $\alpha$-Hölder for any exponent $\alpha \in(0,1)$. Therefore, both projections belong to $A_{\text {loc }}^{2 / \alpha}\left(\partial X_{3} \backslash\{\infty\}\right)$ for any $\alpha<1$, which explains Figure 1.2.(iii). In other words, the $L^{p}$-cohomology in degree one is not sensible to the logarithmic term appearing in (1.1). As we will show later, a well chosen Orlicz-Besov space detects the difference between $\pi_{1}$ and $\pi_{2}$, and we are able to distinguish $\infty$ in case (iii) also.

The quasi-isometry invariance of Orlicz-Besov spaces is not evident at first sight, we show it for a class of Young functions which is sufficient for our purposes in Sections 3 and 4. We deal with localization in Section 5 .

As a by product, finer results regarding QIsom $\left(X_{\alpha}\right)$ are obtained. Let us outline them.

1.1. On the Orlicz cohomology of a hyperbolic complex and its localization. Let $X$ be a finite dimensional simplicial complex with bounded geometry. That is, there exists a constant $\mathrm{N}$ such that any vertex of $X$ is contained in at most $\mathrm{N}$ simplices. Suppose $X$ is equipped with a geodesic distance making each of its simplices isometric to a standard Euclidean simplex. We further assume that $X$ is uniformly contractible: it is contractible and any ball $B(x, r)$ in $X$ is contractible in the ball $B\left(x, r^{\prime}\right)$, for some $r^{\prime} \geq r$ which depends only on $r$. In the hyperbolic case, this condition is not restrictive. 
By a Young function we mean an even convex function $\phi: \mathbb{R} \rightarrow \mathbb{R}_{+}$, with $\phi(0)=0$ and $\lim _{t \rightarrow \infty} \phi(t)=+\infty$. For such a function, we introduce here the Orlicz cohomology, denoted by $\ell^{\phi} H^{\bullet}(X)$, of the complex $X$. It consists on a direct generalization of the ordinary $\ell^{p_{-}}$ cohomology introduced in [BP03, Gro93, Pan89a], where $\phi(t)=\phi_{p}(t):=|t|^{p}$. As in the ordinary case, we show that it is a quasi-isometry invariant of $X$.

Suppose that $X$ is in addition a quasi-starlike Gromov-hyperbolic space. Recall that quasistarlike means that any point of $X$ is at uniformly bounded distance from some geodesic ray. Inspired by the works of Pansu [Pan89a, Pan02, Pan08], and Bourdon-Pajot [BP03], we identify the degree one Orlicz cohomology of $X$ with an Orlicz-Besov functional space on its boundary $\partial X$. To this end, we need to assume a decay condition on the Young function.

Definition 1.1. Let $\phi$ be a Young function. We say that $\phi$ is doubling, if there exist $t_{0}>0$ and $\mathrm{K} \geq 2$ such that $\phi(2 t) \leq \mathrm{K} \phi(t)$ for all $t \in\left[0, t_{0}\right]$.

The doubling condition in the above definition is known in the literature as the $\Delta_{2}(0)$ condition. It admits several equivalent formulations, see for example [RR91, Thm. 3 Ch. 2].

Moreover, suppose that the boundary at infinity $\partial X$ admits a visual metric $\varrho$ which is Ahlfors regular of dimension $Q>0$. That is, the $Q$-dimensional Hausdorff measure $H$ of a ball of radius $r \leq$ diam $\partial X$ is comparable to $r^{Q}$. We refer the reader to BH99, GdlH90, Hei01 for basic background.

In the space of pairs $\partial^{2} X$, consider the measure

$$
d \lambda(\xi, \zeta)=\frac{d H \otimes d H(\xi, \zeta)}{\varrho(\xi, \zeta)^{2 Q}} .
$$

If $u: \partial X \rightarrow \mathbb{R}$ is a measurable function, we define its Orlicz-Besov $\phi$-norm as

$$
\langle u\rangle_{\phi}:=\inf \left\{\alpha>0: \int_{\partial^{2} X} \phi\left(\frac{u(\xi)-u(\zeta)}{\alpha}\right) d \lambda(\xi, \zeta) \leq 1\right\} .
$$

Then, the Orlicz-Besov space is by definition

$$
B^{\phi}(\partial X, \varrho):=\left\{u: \partial X \rightarrow \mathbb{R} \text { measurable }:\langle u\rangle_{\phi}<\infty\right\} .
$$

We denote by $\mathbb{R}$ the functions on $\partial X$ which are constant $H$-almost everywhere. Then the space $B^{\phi}(\partial X, \varrho) / \mathbb{R}$ when equipped with the norm $\langle\cdot\rangle_{\phi}$ is a Banach space.

Theorem 1.2. Let $\phi$ be a doubling Young function, and $\varrho$ be an Ahlfors regular visual metric on $\partial X$. There exists a canonical isomorphism of Banach spaces between $\ell^{\phi} H^{1}(X)$ and $B^{\phi}(\partial X, \varrho) / \mathbb{R}$. In particular, $\ell^{\phi} H^{1}(X)$ is reduced.

Notice that by [BP03, Prop. 2.1], any Ahlfors regular compact metric space $Z$ is biLipschitz homeomorphic to $\partial X$ for some geometric hyperbolic complex $X$ as above, and the quasi-isometry class of $X$ depends only on the quasi-symmetry class of $Z$. In particular, Orlicz-Besov spaces are quasisymmetry invariants of $Z$. When the metric space $Z$ is the Euclidean $n$-sphere, and $\phi=\phi_{p}$, this Orlicz-Besov space coincides with the classical Besov space $B_{p, p}^{n / p}$ [Tri83].

In order to quantify the influence of a point $\xi \in \partial X$ to the nullity of the cohomology spaces, we introduce a local version of the $\ell^{\phi}$-cohomology. This provides us with an interesting tool capable to distinguish local features of the quasiconformal geometry of the boundary. 
Given a quasi-isometric embedding $\iota: Y \rightarrow X$, where $Y$ is another hyperbolic simplicial complex with bounded geometry, one can define the pull-back of cochains $\iota^{*}$, see for example [BP03]. For $\xi \in \partial X$, denote by $\mathcal{Y}(X, \xi)$ the collection of all such quasi-isometric embeddings with $\iota(\partial Y) \subset \partial_{\xi} X$. We define the space of locally $\phi$-integrable $k$-cochains of $X$ (with respect to $\xi$ ) as

$$
\ell_{\mathrm{loc}}^{\phi}\left(X_{k}, \xi\right):=\left\{\tau: X_{k} \rightarrow \mathbb{R}: \iota^{*}(\tau) \in \ell^{\phi}\left(Y_{k}\right), \forall \iota \in \mathcal{Y}(X, \xi)\right\} .
$$

Here $X_{k}$ and $Y_{k}$ denote the set of $k$-simplices of $X$ and $Y$ respectively. We emphasize the fact that the cochains are defined globally, but the integrability condition is "local". As we will show in Section $5 . \ell_{\mathrm{loc}}^{\phi}\left(X_{k}, \xi\right)$ is a Fréchet space and the coboundary operators

$$
\delta_{k}: \ell_{\mathrm{loc}}^{\phi}\left(X_{k}, \xi\right) \rightarrow \ell_{\mathrm{loc}}^{\phi}\left(X_{k+1}, \xi\right)
$$

are Lipschitz continuous.

Definition 1.2. Consider a point $\xi \in \partial X$. We define the local $\ell^{\phi}$-cohomology of $X$ with respect to $\xi$ as

$$
\ell_{\mathrm{loc}}^{\phi} H^{k}(X, \xi):=\operatorname{ker} \delta_{k} / \operatorname{im} \delta_{k-1} .
$$

The reduced local $\ell^{\phi}$-cohomology is defined as usual taking the quotient by $\overline{\operatorname{im} \delta_{k-1}}$.

Notice that by definition, the local $\ell^{\phi}$-cohomology is a quasi-isometry invariant of the pair $(X, \xi)$. More precisely, if $F: X \rightarrow X^{\prime}$ is a quasi-isometry between two hyperbolic simplicial complexes as above, then $\ell_{\mathrm{loc}}^{\phi}(X, \xi)$ is isomorphic as a topological vector space to $\ell_{\mathrm{loc}}^{\phi}\left(X^{\prime}, F(\xi)\right)$. We denote also by $F$ the boundary extension.

As for the global cohomology, we can identify the local Orlicz cohomology in degree one with a local Orlicz-Besov space on the parabolic boundary $\partial_{\xi} X:=\partial X \backslash\{\xi\}$. We suppose that $\partial_{\xi} X$ is equipped with an Ahlfors regular parabolic visual metric $\varrho_{\xi}$. We define the local Orlicz-Besov space on $\partial_{\xi} X$ as

$$
B_{\text {loc }}^{\phi}\left(\partial_{\xi} X, \varrho_{\xi}\right):=\left\{u: \partial_{\xi} X \rightarrow \mathbb{R}:\langle u\rangle_{\phi, \xi, K}<\infty, \forall \text { compact } K \subset \partial_{\xi} X\right\},
$$

where $\langle\cdot\rangle_{\phi, \xi, K}$ is the seminorm defined as in 1.2 , but replacing $\varrho$ by $\varrho_{\xi}$ and integrating over the compact $K$.

Theorem 1.3. Let $\phi$ be a doubling Young function, and $\varrho_{\xi}$ be an Ahlfors regular parabolic visual metric on $\partial_{\xi} X$. There exists a canonical isomorphism of Fréchet spaces between $\ell_{\mathrm{loc}}^{\phi} H^{1}(X, \xi)$ and $B_{\mathrm{loc}}^{\phi}\left(\partial_{\xi} X, \varrho_{\xi}\right) / \mathbb{R}$. In particular, $\ell_{\mathrm{loc}}^{\phi} H^{1}(X, \xi)$ is reduced.

These identifications are very useful to define several quasi-isometry invariants. Consider a family of doubling Young functions $\left\{\phi_{i}: i \in I\right\}$ indexed on a totally ordered set $I$, and suppose that it is non-decreasing in the sense that if $i \leq j$ in $I$, then $\phi_{i} \preceq \phi_{j}$ (see Section 2 for the definition of the relation $\preceq$ ). We define the critical exponent of $I$ as

$$
p_{\neq 0}(X, I):=\inf \left\{i \in I: \ell^{\phi_{i}} H^{1}(X) \neq 0\right\} .
$$

Notice that by Theorem 1.2 there is a canonical inclusion $\ell^{\phi_{1}} H^{1}(X) \subset \ell^{\phi_{2}} H^{1}(X)$ whenever $\phi_{1} \preceq \phi_{2}$. When the family of Young functions is given by $\left\{\phi_{p}: p \in[1,+\infty)\right\}$, this exponent is the well known critical exponent associated to the $\ell_{p}$-cohomology of $X$. An analogous exponent $p_{\neq 0}(X, \xi, I)$ can be defined for the local Orlicz cohomology.

Finer invariants can be given following the ideas of [Bou07, BK13, BK12]. Consider the algebra of continuous Orlicz-Besov functions

$$
A^{\phi}=A^{\phi}\left(\partial X, \varrho_{w}\right):=\left\{u \in B^{\phi}\left(\partial X, \varrho_{w}\right): u \text { is continuous }\right\} .
$$


It is a unital Banach algebra when equipped with the norm $N_{\phi}(u):=\|u\|_{\infty}+\langle u\rangle_{\phi}$. The spectrum of $A^{\phi}$, denoted by $\operatorname{Sp}\left(A^{\phi}\right)$, is a Hausdorff compact topological space invariant by Banach algebra isomorphisms. In particular, the spectrum, as well as its topological dimension $s(\phi):=\operatorname{dim}_{T} \operatorname{Sp}\left(A_{\phi}\right)$, are quasi-isometry invariants of $X$. For instance, given an indexed family $\left\{\phi_{i}: i \in I\right\}$ as before, the function $s: I \rightarrow \mathbb{N} \cup\{\infty\}, i \mapsto s(i)=s\left(\phi_{i}\right)$, provides another quasi-isometry invariant of $X$.

The spectrum of $A_{\phi}$ is a quotient space of $\partial X$, where the equivalence relation is given by

$$
\xi \sim_{\phi} \zeta \text { if, and only if, } u(\xi)=u(\zeta), \forall u \in A_{\phi} .
$$

The $\ell^{\phi}$-equivalence classes provide a partition of $\partial X$ which must be preserved by the boundary homeomorphism of any self quasi-isometry of $X$.

The same considerations can be carried out for the local Orlicz cohomology, by considering the unital Fréchet algebra $A_{\mathrm{loc}}^{\phi}\left(\partial_{\xi} X, \varrho_{\xi}\right)$ of continuous Orlicz-Besov functions on the parabolic boundary $\partial_{\xi} X$. In particular, the boundary homeomorphism of a self quasi-isometry of $X$ which fixes the point $\xi$, must preserve the local $\ell^{\phi}$-cohomology classes of $\partial_{\xi} X$.

1.2. On quasi-isometries of Heintze groups. We will focus on the global and local Orlicz cohomology in degree one of $X_{\alpha}$, for the family of doubling Young functions given by

$$
\phi_{p, \kappa}(t)=\frac{|t|^{p}}{\log \left(e+|t|^{-1}\right)^{\kappa}},(p, \kappa) \in I=[1,+\infty) \times[0,+\infty) .
$$

In order to simplify the notation, we indicate the Orlicz spaces and the norms associated to the functions $\phi_{p, \kappa}$ with the superscript " $p, \kappa$ ". The set of pairs $(p, \kappa) \in I$ is endowed with the lexicographic order, so we obtain a non-decreasing family of Young functions as in the previous section.

We will define a non-decreasing sequence of closed Lie subgroups of $N$,

$$
\{e\}=K_{0} \leq H_{1} \leq K_{1} \leq H_{2} \leq K_{2} \leq \cdots H_{d} \leq K_{d}=N,
$$

whose left cosets will be identified with local cohomology classes for appropriate choices of the parameters $p$ and $\kappa$.

Denote by $\mu_{1} \leq \cdots \leq \mu_{d}$ the distinct eigenvalues of $\alpha$, and let $\mathcal{B}$ be a basis of $\mathfrak{n}$ on which $\alpha$ assumes its Jordan canonical form. In the basis $\mathcal{B}$, we have the decomposition

$$
\alpha=\bigoplus_{i=1}^{d} \bigoplus_{j=1}^{k_{i}} J\left(\mu_{i}, m_{i j}\right) .
$$

Here $J\left(\mu_{i}, m_{i j}\right)$ denotes a Jordan block of size $m_{i j}$ associated to the eigenvalue $\mu_{i}$. Let $V_{i}$ be the generalized eigenspace associated with $\mu_{i}$.

Let $\mathfrak{k}_{0}=W_{0}=\{0\}$, and for each $i \in\{1, \ldots, d\}$, let

$$
W_{i}=\bigoplus_{r=1}^{i} V_{r}, \text { and } \mathfrak{k}_{i}=\operatorname{LieSpan}\left(W_{i}\right)
$$

That is, $\mathfrak{k}_{i}$ is the Lie sub-algebra of $\mathfrak{n}$ generated by $W_{i}$. Define $K_{i}$ to be the closed Lie subgroup of $N$ whose Lie algebra is $\mathfrak{k}_{i}$. Note that the set of left cosets $N / K_{i}$ is a smooth manifold, and the canonical projection is a smooth map.

For each $i \in\{1, \ldots, d\}$, we also let $m_{i}=\max \left\{m_{i j}: 1 \leq j \leq k_{i}\right\}$ be the maximal size of the Jordan blocks with eigenvalue $\mu_{i}$. Consider the $\mu_{i}$-eigenvectors belonging to the Jordan 
blocks of size $m_{i}$, and denote by $V_{i}^{0}$ the vector space their span. Finally, consider $\mathfrak{h}_{i} \leq \mathfrak{k}_{i}$ the Lie subalgebra spanned by $W_{i-1} \oplus V_{i}^{0}$, and $H_{i}$ its corresponding closed Lie subgroup. Notice that $\mathfrak{h}_{i}=\mathfrak{k}_{i}$ only when $m_{i}=1$.

The parabolic boundary $\partial_{\infty} X_{\alpha}$ can be identified with $N$. A left invariant parabolic visual metric $\varrho_{\infty}$ can be defined on $N$, and so that $\tau$ acts as a dilation. From this, one easily checks that $\varrho_{\infty}$ is Ahlfors regular (see Section 6 for more details). In the statement of the next theorem we write $A_{\mathrm{loc}}^{p, \kappa}$ for $A_{\mathrm{loc}}^{p, \kappa}\left(N, \varrho_{\infty}\right)$

Theorem 1.4 (Local classes with respect to $\infty$ ). For each $i \in\{1, \ldots, d\}$, consider the exponent $p_{i}=\operatorname{tr}(\alpha) / \mu_{i}$, and set $p_{d+1}=1$.

(1) Suppose $p \in\left(p_{i+1}, p_{i}\right)$ and $\kappa \geq 0$. The spectrum of $A_{\text {loc }}^{p, \kappa}$ is homeomorphic to $N / K_{i}$.

(2) Suppose $p=p_{i}$ and $m_{i}=1$.

(a) If $\kappa \leq 1$, the spectrum of $A_{\mathrm{loc}}^{p, \kappa}$ is homeomorphic to $N / K_{i}$.

(b) If $\kappa>1$, the spectrum of $A_{\mathrm{loc}}^{p, \kappa}$ is homeomorphic to $N / K_{i-1}$.

(3) Suppose $p=p_{i}, \kappa \in\left(1+p_{i}\left(m_{i}-2\right), 1+p_{i}\left(m_{i}-1\right)\right]$, and $m_{i} \geq 2$. The spectrum of $A_{\mathrm{loc}}^{p, \kappa}$ is homeomorphic to $N / H_{i}$.

In particular, in all cases, the $(p, \kappa)$-local cohomology classes on $N$ coincide with the lefts cosets of the corresponding subgroup.

Let $i(\alpha)=\min \left\{i: K_{i}=N\right\}$. Then the local critical exponent satisfies

$$
\left(p_{i(\alpha)}, 0\right) \leq p_{\neq 0}\left(X_{\alpha}, \infty, I\right) \leq\left(p_{i(\alpha)}, 1+p_{i(\alpha)}\left(m_{i(\alpha)}-2\right)^{+}\right),
$$

where $a^{+}=\max \{a, 0\}$.

The picture is quite different for the local cohomology with respect to the points of $N$.

Theorem 1.5 (Local cohomology with respect to $\xi \in N)$. The local $\ell^{p, \kappa}$-cohomology of $X_{\alpha}$ with respect to a point $\xi \in N$ is trivial if, and only if, $(p, \kappa) \leq\left(p_{1}, 1+p_{1}\left(m_{1}-1\right)\right)$.

In particular, $p_{\neq 0}\left(X_{\alpha}, \xi, I\right)=\left(p_{1}, 1+p_{1}\left(m_{1}-1\right)\right)$ for any $\xi \in N$. Theorem 1.1 follows therefore from Theorems 1.4 and 1.5, see Section 6. Notice that in the Carnot type case, $p_{\neq 0}\left(X_{\alpha}, \infty, I\right)=p_{\neq 0}\left(X_{\alpha}, \xi, I\right)=\left(p_{1}, 1\right)$ for any $\xi \in N$. As an immediate consequence, we obtain the following result for the global cohomology.

Corollary 1.6. The critical exponent of the $\ell^{p, \kappa}$-cohomology of $X_{\alpha}$ is given by

$$
p_{\neq 0}\left(X_{\alpha}, I\right)=\left(p_{1}, 1+p_{1}\left(m_{1}-1\right)\right) .
$$

Moreover, the $\ell^{p, \kappa}$-cohomology is also trivial at this critical exponent.

As an example, consider the Heintze group $X_{3}$ introduced at the beginning of this section. The critical exponents are in this case is $p_{\neq 0}\left(X_{3}, \infty, I\right)=(2,3)$ and $p_{\neq 0}\left(X_{3}, \xi, I\right)=(2,1)$ for any $\xi \in \mathbb{R}^{2}$. The subgroup $H_{1}$ is $\mathbb{R} \times\{0\}$. Notice that even though the conformal dimension of $\partial X_{3}$ is equal to 2 , it is not attained. This is proved in [HP11][Thm. 1.8] by techniques of two dimensional conformal dynamics which do not apply to the higher dimensional case. We refer the reader to [MT10] for an account on conformal dimension.

The $\kappa$-coordinate of the critical exponent can be interpreted as a second order quasiisometry invariant "dimension" of a hyperbolic complex $X$ as above. When the Ahlfors regular conformal dimension $Q$ of $\partial X$ is attained, the critical exponents satisfy the inequality $p_{\neq 0}(X, \xi, I) \leq(Q, 1)$ for any $\xi \in \partial X$, see Lemma 5.2 . 
Corollary 1.7. If $m_{1} \geq 2$, the Ahlfors regular conformal dimension of $\partial X_{\alpha}$ is not attained.

The pointed sphere conjecture is not settled in the Carnot type case. Our methods do not apply, essentially, because Carnot groups equipped with Carnot-Carathéodory metrics are Loewner spaces [Hei01]. In particular, they contain "a lot of rectifiable curves", which makes difficult to distinguish points by invariants strongly related to the moduli of curves.

The restrictions imposed on self quasi-isometries of $X_{\alpha}$ preserving a foliation at infinity are manifest in the rigidity results due to Xie, in the case when $N$ is abelian or isomorphic to a Heisemberg group. It is there shown that self quasi-isometries are almost-isometries, i.e. a $(1, \mathrm{C})$-quasi-isometry. This question is motivated by the work of Farb and Mosher on abelian-by-cyclic groups [FM00]. We apply Xie's approach to our more general context.

Corollary 1.8. Let $X_{\alpha}$ be a purely real Heintze group, and suppose that the normalizer of $\mathfrak{h}_{1}, N_{\mathfrak{n}}\left(\mathfrak{h}_{1}\right)=\left\{v \in \mathfrak{n}:[v, w] \in \mathfrak{h}_{1}, \forall w \in \mathfrak{h}_{1}\right\}$, is strictly bigger than $\mathfrak{h}_{1}$. Then, any self quasi-isometry of $X_{\alpha}$ is an almost isometry.

The Corollary 1.8 applies, in particular, in the case when $N$ is abelian or isomorphic to a Heinsemberg group, and $X_{\alpha}$ is not of Carnot type, generalizing therefore the previous known results.

We obtain also results regarding the quasi-isometric classification of Heintze groups. It is conjectured that two purely real Heintze groups are quasi-isometric if, and only if, they are isomorphic [Ham87, Cor12]. By [Pan89c, Theorem 2], the conjecture is true when $X_{\alpha}$ and $X_{\beta}$ are both purely real Heintze groups of Carnot type. Notice that if $\alpha_{1}$ is the restriction of $\alpha$ to $\mathfrak{h}_{1}$, then $H_{1} \rtimes_{\alpha_{1}} \mathbb{R}$ is a Heintze group of Carnot type. Also, that if there exists a quasi-isometry between two Heintze groups $X_{\alpha}$ and $X_{\beta}$, then there exists a quasi-isometry sending $\infty$ to $\infty$ Cor12, Lemma 6.D.1]. From Pansu's theorem and Theorem 1.4, we obtain the following consequence.

Corollary 1.9. Let $X_{\alpha}$ and $X_{\beta}$ be two purely real Heintze groups. If they are quasiisometric, then $H_{1}^{(\alpha)} \rtimes_{\alpha_{1}} \mathbb{R}$ and $H_{1}^{(\beta)} \rtimes_{\beta_{1}} \mathbb{R}$ are isomorphic. In particular, if $X_{\alpha}$ is of Carnot type and $X_{\beta}$ is not, then they are not quasi-isometric.

In the abelian type case, that is, when $N$ is abelian, the algebra is not playing any role, and we obtain another, and more direct, proof of the following result of Xie.

Corollary 1.10. [Xie14a, Theorem 1.1] Let $X_{\alpha}$ and $X_{\beta}$ be two purely real Heintze groups of abelian type. If they are quasi-isometric, there exists $\lambda>0$ such that $\alpha$ and $\lambda \beta$ have the same Jordan form. In particular, $X_{\alpha}$ and $X_{\beta}$ are isomorphic.

We refer the reader to [Cor12, Section 6.B] for more details about the quasi-isometric classification of Heintze groups. Notice that Theorem 1.4 provides new invariants related to the sizes of the Jordan blocks of $\alpha$. We were not able to compute the spectrum of $A_{\text {loc }}^{p, \kappa}$ for all the possible values of $(p, \kappa)$. One could expect to show, by a more refined analysis, that the Jordan form of the derivation $\alpha$, up to scalar multiplication, is a quasi-isometry invariant of the Heintze group, generalizing thus the first conclusion of Corollary 1.10.

1.3. Notations and conventions. To make the notation clearer we will write the constants in sans-serif font, e.g. C, K, etc.. If $f$ and $g$ are non-negative real functions defined on a set $A$, we say that $f \lesssim g$ if there exists a constant C, such that $f(a) \leq \mathrm{C} g(a)$ for all $a \in A$. If both inequalities are true, $f \lesssim g$ and $g \lesssim f$, we say that $f$ and $g$ are comparable and we denote it by $f \asymp g$. 
Acknowledgments. I would like to specially thanks Pierre Pansu for all his help and advice during the realization of this work, and for explaining me his works on $L^{p}$-cohomology. I am also particularly indebted to Romain Tessera for sharing with me his ideas about Orlicz spaces and their applications to Heintze groups. I also thanks Yves de Cornulier for helpful discussions on quasi-isometries of Heintze groups. This work was supported by the ANR project "GDSous/GSG" no. 12-BS01-0003-01.

\section{Preliminaries on the theORY of ORLICZ SPACES}

2.1. Generalities. In this section we recall some basic facts about Orlicz spaces which will be used throughout this articles. We refer to [RR91 for a general treatment. Recall that a Young function is an even convex function $\phi: \mathbb{R} \rightarrow \mathbb{R}_{+}$which satisfies $\phi(0)=0$, and $\lim _{t \rightarrow \infty} \phi(t)=+\infty$. Note that we require $\phi$ to be finite valued, so in particular, $\phi$ is locally Lipschitz. We will also assume that $\phi(t) \neq 0$ if $t \neq 0$.

Any Young function can be represented as an integral

$$
\phi(t)=\int_{0}^{|t|} \phi^{\prime}(s) d s,
$$

where $\phi^{\prime}: \mathbb{R}_{+} \rightarrow \mathbb{R}_{+}$is nondecreasing left continuous and $\phi^{\prime}(0)=0$ [RR91, Cor. 2 Ch. 1]. The function $\phi^{\prime}$ coincides with the derivative of $\phi$ except perhaps for at most a countable number of points.

Let the space $\Omega$ be given with a $\sigma$-algebra and a $\sigma$-finite measure $\mu$. For any measurable function $f$ on $\Omega$, the Luxembourg norm of $f$ is defined as

$$
\|f\|_{\phi}=\inf \left\{\alpha>0: \int_{\Omega} \phi\left(\frac{f}{\alpha}\right) d \mu \leq 1\right\} \in[0,+\infty]
$$

where it is understood that $\inf (\emptyset)=+\infty$. The Orlicz space $L^{\phi}(\Omega, \mu)$ is the vector space of measurable functions $f$ on $\Omega$ such that $\|f\|_{\phi}<\infty$ [RR91, Thm. 10 Ch. 3]. Up to almost everywhere null functions, $L^{\phi}(\Omega, \mu)$ is a Banach space with the norm $\|\cdot\|_{\phi}$. As usual, we simply write $\ell^{\phi}(\Omega)$ when $\mu$ is the counting measure.

If $\mathrm{K} \geq 1$ is any constant, the identity map $L^{\mathrm{K} \phi}(\Omega, \mu) \rightarrow L^{\phi}(\Omega, \mu)$ is continuous and bijective, and therefore, by the open mapping theorem, $\|\cdot\|_{\kappa_{\phi}}$ and $\|\cdot\|_{\phi}$ are equivalent norms:

$$
\exists \mathrm{C}=\mathrm{C}(\mathrm{K}, \phi) \text { s.t. }\|\cdot\|_{\phi} \leq\|\cdot\|_{\mathrm{K} \phi} \leq \mathrm{C}\|\cdot\|_{\phi} .
$$

Let us point out that when the measure $\mu$ is finite, the Orlicz space $L^{\phi}(\Omega, \mu)$ is contained in $L^{1}(\Omega, \mu)$ and the inclusion is continuous [RR91, Cor. 3 Ch. 1]. In particular, in locally compact spaces equipped with a regular measure, Orlicz integrable functions are locally integrable.

We will not need the Hölder inequality in this paper, but let us remark that it holds exactly as in the ordinary case by considering $\psi: \mathbb{R} \rightarrow[0,+\infty]$ the convex conjugate of $\phi$ [RR91, Thm. 3 Ch. 1]. This inequality serves also to define the Orlicz spaces by means of the so called Orlicz norm instead of the Luxembourg norm.

The main difficulty in dealing with the norm $\|\cdot\|_{\phi}$ is that it is not comparable to the function

$$
f \mapsto \phi^{-1}\left(\int_{\Omega} \phi(f) d u\right)
$$


This is only the case when $\phi$ is equivalent to an ordinary power function $\phi_{p}, p \geq 1$. Nevertheless, we will be able to avoid this difficulty by applying Jensen's inequality in order to exchange $\phi$ and an integral or a sum symbol.

2.2. Decay conditions. For the Orlicz spaces considered here, only the decay properties of $\phi$ for small values of $t$ will be relevant for us. Roughly speaking, we do not want our Young functions to be too small near zero. This is precisely the meaning of Definition 1.1. and we will mainly work in this article with doubling Young functions.

Here are some important features of Orlicz spaces of doubling Young functions. First, notice that since $\phi^{\prime}$ is non-decreasing, we have

$$
\frac{t \phi^{\prime}(t)}{\phi(t)} \geq 1 \text { for all } t>0 \text {. }
$$

A useful way to check the doubling property is by considering the exponent

$$
p_{\phi}=\limsup _{t \rightarrow 0} \frac{t \phi^{\prime}(t)}{\phi(t)} \in[1,+\infty] .
$$

Then, $\phi$ is doubling if and only if $p_{\phi}<+\infty$ [RR91, Cor. 4 Ch. 2]. It is important to note that when $\phi$ is doubling, then

$$
f \in L^{\phi}(\Omega, \mu) \text { if, and only if, } \int_{\Omega} \phi(f) d \mu<\infty,
$$

see [RR91, Thm. 2 Ch. 3]. Moreover, when $\phi$ is doubling, if $\left\{f, f_{n}: n \in \mathbb{N}\right\}$ is a sequence in $L^{\phi}(\Omega, \mu)$, then

$$
\left\|f_{n}-f\right\|_{\phi} \rightarrow 0 \text { if, and only if, } \int_{\Omega} \phi\left(f_{n}-f\right) d u \rightarrow 0,
$$

see [RR91, Thm. 12, Ch.3]. In particular, when $\mu$ is a Radon (resp. volume) measure on a locally compact Hausdorff space (resp. Riemannian manifold) $\Omega$, then the usual approximation by continuous (resp. smooth) functions in the $\|\cdot\|_{\phi}$ norm holds.

A doubling Young function has polynomial decay with exponent $p$ for any $p>p_{\phi}$. That is, there exist $t_{0}>0$, and $\mathrm{c}>0$, such that $\phi(t) \geq \mathrm{c} t^{p}$ for $t \in\left[0, t_{0}\right]$. In general, the converse is not true. We also remark that when $\phi$ is doubling of constant $\mathrm{K}$, then $t \phi^{\prime}(t) \leq \mathrm{K} \phi(t)$ for all $t \in\left[0, t_{0}\right]$.

All these conditions are imposed for small values of $t$. When dealing with discrete Orlicz spaces, the behavior of $\phi$ at infinity is essentially irrelevant. To justify this, let us introduce the following equivalence relation among Young functions.

Define $\phi_{1} \preceq \phi_{2}$ if there are constants $\mathrm{a}, \mathrm{b} \in \mathbb{R}_{+}$and $t_{0}>0$, so that $\phi_{2}(t) \leq \mathrm{a} \phi_{1}(\mathrm{~b} t)$ for $t \leq t_{0}$. We say that $\phi_{1} \sim \phi_{2}$ for small $t$, if $\phi_{1} \preceq \phi_{2}$ and $\phi_{2} \preceq \phi_{1}$.

Lemma 2.1. Suppose that $\Omega$ is a countable set, and let $\phi_{i}, i=1,2$, be a pair of equivalent Young functions for small $t$. Then the norms $\|\cdot\|_{\phi_{1}}$ and $\|\cdot\|_{\phi_{2}}$ are equivalent.

In particular, given a doubling Young function $\phi$, applying Lemma 2.1 if necessary, we can change $\phi$ by an equivalent (for small $t$ ) Young function, so that the doubling condition 1.1 is satisfied with $t_{0}=+\infty$.

The following lemma will be used later in Section 4 .

Lemma 2.2. Let $\phi$ be a doubling Young function with constants $\mathrm{K}$ and $t_{0}=+\infty$. Then for all $y \in \mathbb{R}_{+}$and $x \in[0,1]$ we have $x^{\mathrm{K}} \phi(y) \leq \phi(x y)$. 
Proof. For each $x \in[0,1]$, define the function $\tau_{x}: \mathbb{R}_{+} \rightarrow \mathbb{R}_{+}$by $\tau_{x}(y)=\phi(x y) / \phi(y)$. Notice that $\tau_{x}(y) \leq 1$ because $x \leq 1$ and $\phi$ is increasing. Since $t \phi^{\prime}(t) \leq \phi(t)$ for all $t \in \mathbb{R}_{+}$, we have

$$
\log \left(\frac{1}{\tau_{x}(y)}\right)=\int_{x y}^{y} \frac{\phi^{\prime}(t)}{\phi(t)} d t \leq \int_{x y}^{y} \frac{\mathrm{K}}{t} d t=\log \left(\frac{1}{x^{\mathrm{K}}}\right) .
$$

That is, $\tau_{x}(y) \geq x^{\mathrm{K}}$. This finishes the proof.

\section{ORLiCZ COHOMOLOGY AND QUASI-ISOMETRY INVARIANCE}

In this section, we extend the classical notion of $L^{p}$-cohomology by considering the larger class of Orlicz spaces. It can be defined as a simplicial, coarse, or De Rham cohomology, depending on the structure of the space under consideration. For our later applications in Section 6, it will be more natural to work with differential forms. Nevertheless, the quasi-isometry invariance is easier to prove in the simplicial context.

In contrast to the $L^{p}$ case, the identification of the discrete and continuous cohomologies is less clear for a general Young function. We will prove it for the degree one cohomology, by using the coarse definition as an intermediary (see Section 3.2).

3.1. The simplicial Orlicz chomology. We first prove the quasi-isometry invariance of the simplicial Orlicz cohomology. It is remarkable that this result is true for any Young function $\phi$, and therefore, in great generality. The proof follows the same lines as the classical proof for the $\ell^{p}$ case, see [BP03].

Let $X$ be a finite dimensional, uniformly contractible, simplicial complex with bounded geometry, see the Introduction for the definition. We denote the geodesic distance on $X$ by $|\cdot-\cdot|$. For $k \in \mathbb{N}$, let $X_{k}$ be the set of $k$-simplices of $X$, and let $C_{k}(X)$ be the vector space of $k$-chains on $X$, i.e. finite real linear combinations of the elements in $X_{k}$.

We will use the following notations: if $c$ is a chain in $X$, the length of $c$, denoted by $\ell(c)$, is the number of $\operatorname{simplices}$ in $\operatorname{supp}(c),\|c\|_{\infty}=\max |c(\sigma)|$, and $\|c\|_{1}=\sum|c(\sigma)|$. Note that our assumptions imply that there exists a function $\mathrm{N}_{X}:[0,+\infty) \rightarrow \mathbb{N}$ such that any ball of radius $r \geq 0$ contains at most $\mathrm{N}_{X}(r)$ simplices. We write $\mathrm{N}=\mathrm{N}_{X}(1)$ for short.

Let $\phi$ be a Young function. The $k$-th space of $\phi$-integrable cochains of $X$ is by definition the Banach space $\ell^{\phi}\left(X_{k}\right)$; i.e. the space of functions $\omega: X_{k} \rightarrow \mathbb{R}$ such that

$$
\|\omega\|_{\phi}:=\inf \left\{\alpha>0: \sum_{\sigma \in X_{k}} \phi\left(\frac{\omega(\sigma)}{\alpha}\right) \leq 1\right\}<\infty .
$$

The standard coboundary operator $\delta_{k}: \ell^{\phi}\left(X_{k}\right) \rightarrow \ell^{\phi}\left(X_{k+1}\right)$ is defined by duality: for $\omega \in \ell^{\phi}\left(X_{k}\right)$ and $\sigma \in X_{k+1}$, let $\delta_{k}(\omega)(\sigma)=\omega(\partial \sigma)$.

By the convexity of $\phi$, and the bounded geometry assumption, $\delta_{k}$ is well defined and is a bounded operator. The proof relies on the following argument which will be used several times in this article. Let us explain it in detail.

Let $\omega \in \ell^{\phi}\left(X_{k}\right)$ and $\sigma \in X_{k+1}$, and suppose that $\partial \sigma \neq 0$. Note that for any $\alpha>0$, we have by convexity

$$
\phi\left(\frac{\delta_{k}(\omega)(\sigma)}{\alpha \mathrm{N}}\right) \leq \phi\left(\frac{\delta_{k}(\omega)(\sigma)}{\alpha\|\partial \sigma\|_{1}}\right) \leq \frac{1}{\|\partial \sigma\|_{1}} \sum_{\sigma^{\prime} \in \operatorname{supp}(\partial \sigma)} \phi\left(\frac{\omega\left(\sigma^{\prime}\right)}{\alpha}\right) .
$$


Summing over $\sigma \in X_{k+1}$, we obtain

$$
\sum_{\sigma \in X_{k+1}} \phi\left(\frac{\delta_{k}(\omega)(\sigma)}{\alpha \mathrm{N}}\right) \leq \mathrm{N} \sum_{\sigma^{\prime} \in X_{k}} \phi\left(\frac{\omega\left(\sigma^{\prime}\right)}{\alpha}\right) .
$$

By (2.1), $\|\omega\|_{\mathrm{N} \phi} \lesssim\|\omega\|_{\phi}<\infty$. In particular, the set of $\alpha>0$ such that the sum on the right hand side is bounded from above by $\mathrm{N}^{-1}$ is not empty, and we can take $\alpha$ arbitrarily close to $\|\omega\|_{\mathrm{N} \phi}$. For such an $\alpha$, we have

$$
\sum_{\sigma \in X_{k+1}} \phi\left(\frac{\delta_{k}(\omega)(\sigma)}{\alpha \mathrm{N}}\right) \leq 1,
$$

which implies $\left\|\delta_{k}(\omega)\right\|_{\phi} \leq \alpha$. Taking infimum, we get $\left\|\delta_{k}(\omega)\right\|_{\phi} \leq \mathrm{N}\|\omega\|_{\mathrm{N} \phi}$. Therefore, $\left\|\delta_{k}(\omega)\right\|_{\phi} \lesssim\|\omega\|_{\phi}$, where the multiplicative constant depends only on $\mathrm{N}$ and $\phi$.

Definition 3.1. The $k$-th $\ell^{\phi}$-cohomology space of $X$ is by definition the topological vector space

$$
\ell^{\phi} H^{k}(X):=\operatorname{Ker} \delta_{k} / \operatorname{Im} \delta_{k-1} .
$$

The reduced cohomology is defined by taking the quotient by $\overline{\operatorname{Im} \delta_{k-1}}$; it is a Banach space.

We focus now on the quasi-isometry invariance of the $\ell^{\phi}$-cohomology spaces. Recall that a function $F: X \rightarrow Y$ between two metric spaces is:

(1) quasi-Lipschitz if there exist constants $\Lambda \geq 1$ and $C \geq 0$ such that

$$
\forall x, y \in X,|F(x)-F(y)| \leq \Lambda|x-y|+\mathrm{C} .
$$

(2) uniformly proper if there exists a function $D_{F}: \mathbb{R}_{+} \rightarrow \mathbb{R}_{+}$such that for any ball $B(y, r)$ in $Y$, we have $\operatorname{diam}\left(F^{-1}(B(y, r))\right) \leq \mathrm{D}_{F}(r)$.

(3) a quasi-isometry if it is quasi-Lipschitz and there exists a quasi-Lipschitz function $G: Y \rightarrow X$ such that $G \circ F$ and $F \circ G$ are at bounded distance from the identity.

We can now state the main result of this section.

Theorem 3.1 (Quasi-isometry invariance). Let $\phi$ be any Young function, and $X, Y$ be two uniformly contractible simplicial complexes with bounded geometry.

(1) Any quasi-Lipschitz uniformly proper function $F: X \rightarrow Y$ induces continuous linear maps $F^{*}: \ell^{\phi} H^{\bullet}(Y) \rightarrow \ell^{\phi} H^{\bullet}(X)$.

(2) If $F, G: X \rightarrow Y$ are two quasi-Lipschitz uniformly proper functions at bounded distance, then $F^{*}=G^{*}$.

(3) If $F: X \rightarrow Y$ is a quasi-isometry, then $F^{*}$ is an isomorphism of topological vector spaces.

The same statements hold for the reduced $\ell^{\phi}$-cohomology.

The proof relies on the following two key lemmas which are proved in [BP03, Section 1]. They serve to define pull-backs and homotopies from quasi-isometries.

Lemma 3.2 ([BP03]). Let $X$ and $Y$ be two uniformly contractible simplicial complexes with bounded geometry. Any quasi-Lipschitz uniformly proper function $F: X \rightarrow Y$ induces maps $c_{F}: X_{\bullet} \rightarrow C_{\bullet}(Y)$ verifying the following conditions:

(1) $c_{F}$ commutes with the boundary operator, i.e. $c_{F}(\partial \sigma)=\partial c_{F}(\sigma)$, and 
(2) for each $k \in \mathbb{N}$, there are constants $\mathrm{N}_{k}$ and $\mathrm{L}_{k}$, depending only on $k$ and the geometric data of $X, Y$ and $F$, such that

$$
\left\|c_{F}(\sigma)\right\|_{\infty} \leq \mathrm{N}_{k}, \text { and } \ell\left(c_{F}(\sigma)\right) \leq \mathrm{L}_{k}
$$

The map $c_{F}: X_{\bullet} \rightarrow C_{\bullet}(Y)$ is constructed by induction on $k$. For $k=0$ and $\sigma \in X_{0}, c_{F}(\sigma)$ is defined to be any vertex of $Y$ at uniformly bounded distance from $F(\sigma)$. The induction can be carried out since $X$ is uniformly contractible.

Lemma 3.3 ([BP03]). Let $F, G: X \rightarrow Y$ be two quasi-Lipschitz uniformly proper functions at bounded uniform distance. Then there exists a homotopy $h: X_{\bullet} \rightarrow C_{\bullet+1}(Y)$ between $c_{F}$ and $c_{G}$. That is, a map verifying

(i) for $\sigma \in X_{0}, \partial h(\sigma)=c_{F}(\sigma)-c_{G}(\sigma)$, and

(ii) for $\sigma \in X_{k}, k \geq 1, \partial h(\sigma)+h(\partial \sigma)=c_{F}(\sigma)-c_{G}(\sigma)$.

As before, $\|h(\sigma)\|_{\infty}$ and $\ell(h(\sigma))$ are uniformly bounded on $X_{k}$ by constants $\mathrm{N}_{k}^{\prime}$ and $\mathrm{L}_{k}^{\prime}$, depending only on the geometric data of $X, Y, F$ and $G$.

The homotopy $h$ is also defined by induction on $k$. For $k=0$ and $\sigma \in X_{0}, h(\sigma)$ is defined to be any 1-chain in $Y$ verifying

$$
\partial h(\sigma)=c_{F}(\sigma)-c_{G}(\sigma), \ell(h(\sigma)) \lesssim\left|c_{F}(\sigma)-c_{G}(\sigma)\right|, \text { and }\|h(\sigma)\|_{\infty}=1 .
$$

Proof of Theorem 3.1. Let us prove (1). For $\omega \in \ell^{\phi}\left(Y_{k}\right)$, define the pull-back

$$
F^{*}(\omega): X_{k} \rightarrow \mathbb{R}, F^{*}(\omega)(\sigma)=\omega\left(c_{F}(\sigma)\right), \sigma \in X_{k} .
$$

We show that $F^{*}(\omega)$ belongs to $\ell^{\phi}\left(X_{k}\right)$. The proof will also show the continuity of $F^{*}$. For $\alpha>0$, by convexity of $\phi$, we have

$$
\sum_{\sigma \in X_{k}} \phi\left(\frac{F^{*}(\omega)(\sigma)}{\mathrm{N}_{k} \mathrm{~L}_{k} \alpha}\right) \leq \sum_{\sigma \in X_{k}} \sum_{\sigma^{\prime} \in \operatorname{supp} c_{F}(\sigma)} \phi\left(\frac{\omega\left(\sigma^{\prime}\right)}{\alpha}\right) .
$$

For fixed $\sigma^{\prime} \in Y_{k}$, the uniform properness of $F$ implies that the set of $\sigma \in X_{k}$ for which $\sigma^{\prime} \in \operatorname{supp} c_{F}(\sigma)$, is contained in a ball of $X$ of radius $r=\mathrm{D}_{F}\left(2 \mathrm{~L}_{k}+\Lambda+\mathrm{C}\right)$. Then, their number is bounded by $\mathrm{N}_{X}(r)$. This implies

$$
\sum_{\sigma \in X_{k}} \sum_{\sigma^{\prime} \in \operatorname{supp} c_{F}(\sigma)} \phi\left(\frac{\omega\left(\sigma^{\prime}\right)}{\alpha}\right) \leq \mathrm{N}_{X}(r) \sum_{\sigma^{\prime} \in Y_{k}} \phi\left(\frac{\omega\left(\sigma^{\prime}\right)}{\alpha}\right) .
$$

As in $(3.2)$, this gives $\left\|F^{*}(\omega)\right\|_{\phi} \leq \mathrm{N}_{k} \mathrm{~L}_{k}\|\omega\|_{\left(\mathrm{N}_{X}(r) \phi\right)} \lesssim\|\omega\|_{\phi}$.

Denote by $\delta_{k}^{X}$ and $\delta_{k}^{Y}$ the coboundary operators of $X$ and $Y$ respectively. Then $\delta_{k}^{X} \circ F^{*}=$ $F^{*} \circ \delta_{k}^{Y}$, since $c_{F}$ commutes with the boundary operator. Thus, $F^{*}$ induces a continuous linear map $F^{*}: \ell^{\phi} H^{k}(Y) \rightarrow \ell^{\phi} H^{k}(X)$. This also holds for the reduced cohomology by the continuity of $F^{*}$.

Let us prove (2). Suppose that $\|F-G\|_{\infty}<\infty$. For $\omega \in \ell^{\phi}\left(Y_{k+1}\right)$, define the pull-back

$$
h^{*}(\omega): X_{k} \rightarrow \mathbb{R}, h^{*}(\omega)(\sigma)=\omega(h(\sigma)), \sigma \in X_{k} .
$$

As in the proof of (1), by the convexity of $\phi$, we have

$$
\sum_{\sigma \in X_{k}} \phi\left(\frac{h^{*}(\omega)(\sigma)}{\mathrm{N}_{k}^{\prime} \mathrm{L}_{k}^{\prime} \alpha}\right) \leq \sum_{\sigma \in X_{k}} \sum_{\sigma^{\prime} \in \operatorname{supp} h(\sigma)} \phi\left(\frac{\omega\left(\sigma^{\prime}\right)}{\alpha}\right) .
$$

For fixed $\sigma^{\prime} \in Y_{k+1}$, the set of $\sigma \in X_{k}$ for which $\sigma^{\prime} \in \operatorname{supp} h(\sigma)$, is contained in a ball of radius $r^{\prime}$ which depends only on $\mathrm{L}_{k}^{\prime}$ and the geometric data of $X, Y, F$ and $G$. 
Therefore, as in (1), we obtain $\left\|h^{*}(\omega)\right\|_{\phi} \leq \mathrm{N}_{k}^{\prime} \mathrm{L}_{k}^{\prime}\|\omega\|_{\left(\mathrm{N}_{X}\left(r^{\prime}\right) \phi\right)} \lesssim\|\omega\|_{\phi}$. This shows that $h^{*}: \ell^{\phi}\left(Y_{k+1}\right) \rightarrow \ell^{\phi}\left(X_{k}\right)$ is a bounded operator. By Lemma 3.3 , we have

$$
h^{*} \circ \delta_{k}^{Y}+\delta_{k-1}^{X} \circ H^{*}=F^{*}-G^{*},
$$

which implies that $F^{*}$ and $G^{*}$ induce the same map in $\ell^{\phi}$-cohomology.

Finally, (3) results from (2) since $F^{*} \circ G^{*}$ and $G^{*} \circ F^{*}$ are the identity maps.

3.2. The degree one coarse Orlicz cohomology. Let $(X, d)$ be a complete proper metric space equipped with a Radon measure $\mu$. We say that $X$ has bounded geometry if

$$
0<v(r)=\inf _{x \in X}\{\mu(B(x, r))\} \leq V(r)=\sup _{x \in X}\{\mu(B(x, r))\}<\infty, \forall r>0 .
$$

We say that $X$ has the midpoint property if there exists a constant $c_{X} \geq 0$ such that for all $x, y \in X$, there is some $z \in X$ with

$$
\max \{d(z, x), d(z, y)\} \leq \frac{d(x, y)}{2}+\mathrm{c}_{X} .
$$

This property is satisfied for instance, if $X$ is $(1, \mathrm{c})$-quasi-isometric to a geodesic metric space, with a mid-point constant equal to $3 \mathrm{c}$. In the rest of this section we suppose that $X$ has bounded geometry and satisfies the mid-point property. This and the next section are inspired by [Pan89a, Shc14] and we will closely follow the ideas there exposed.

A kernel in $X$ is a bounded function $\kappa: X \times X \rightarrow \mathbb{R}_{+}$such that

(1) for all $x \in X$,

$$
\int_{X} \kappa(x, y) d \mu(y)=1, \text { and }
$$

(2) there are constants $\varepsilon>5 \mathrm{c}_{X}, \delta>0$ and $R>0$ such that

$$
\kappa(x, y) \geq \delta \text { if } d(x, y) \leq \varepsilon \text { and } \kappa(x, y)=0 \text { if } d(x, y) \geq R .
$$

Such a kernel always exists, consider for example

$$
\kappa(x, y)=\frac{1}{\mu(B(x, r))} \mathbb{1}_{\{d(x, y)<r\}}\left(\text { with } r>5 c_{X}\right) .
$$

The positivity radius of a kernel $\kappa$ is the best constant $\varepsilon_{\kappa}$ for which (2) is verified. The convolution of two kernels $\kappa_{1}$ and $\kappa_{2}$ is the kernel given by

$$
\kappa_{1} * \kappa_{2}(x, y)=\int_{X} \kappa_{1}(x, z) \kappa_{2}(z, y) d \mu(z) .
$$

Notice that by the definition of $\mathrm{c}_{X}$, the positivity radius of self convolutions of a kernel $\kappa$ satisfies

$$
\varepsilon_{\kappa^{* 2}} \geq \frac{6}{5} \varepsilon_{\kappa}, \text { and in particular, } \varepsilon_{\kappa^{* 2 m}} \rightarrow+\infty \text { when } m \rightarrow+\infty .
$$

This implies that given any two kernels $\kappa_{1}$ and $\kappa_{2}$ on $X$, there is some $m \in \mathbb{N}$ and some constant C (depending only on the kernels) such that

$$
\kappa_{1} \leq \mathrm{C} \kappa_{2}^{* 2^{m}} \text {. }
$$

Given a kernel $\kappa$, we denote by $\mu_{\kappa}$ the measure $\kappa d \mu \otimes d \mu$ on $X^{2}=X \times X$. The space of $(\phi, \kappa)$-integrable cocycles on $X$ is the closed subspace of $L^{\phi}\left(X^{2}, \mu_{\kappa}\right)$ given by the measurable functions $\omega: X \times X \rightarrow \mathbb{R}$ verifying $\omega(x, y)=\omega(x, z)+\omega(z, y)$ for almost every $x, y, z \in X$. We denote by $Z_{\phi, \kappa}(X)$ the Banach space of $(\phi, \kappa)$-integrable cocycles on $X$. We write $\|\cdot\|_{\phi, \kappa}$ for the corresponding Luxembourg norm of a cocycle. 
Given a measurable function $u: X \rightarrow \mathbb{R}$, we can define the cocycle $D u(x, y)=u(x)-u(y)$. We obtain in this way a bounded operator $D: L^{\phi}(X, \mu) \rightarrow Z_{\phi, \kappa}(X)$. We define the coarse $L_{\kappa}^{\phi}$-cohomology in degree one as the quotient $L_{\kappa}^{\phi} H^{1}(X):=Z_{\phi, \kappa}(X) / \operatorname{Im}(\mathrm{D})$.

Observe that by (3.5), different kernels define equivalent $\phi$-norms when restricted to cocycles. That is, the integrability condition is independent of the kernel. Notice also that a measurable function $u: X \rightarrow \mathbb{R}$ with $\|D u\|_{\phi, \kappa}<\infty$ is in $L_{\text {loc }}^{1}(X)$.

We need to define the convolution of functions and cocylces with respect to a kernel $\kappa$. Given a measurable function $u: X \rightarrow \mathbb{R}$, we define the convolution

$$
u * \kappa(x)=\int_{X} u(z) \kappa(x, z) d \mu(z),
$$

and given a cocycle $\omega: X \times X \rightarrow \mathbb{R}$, we define the convolution

$$
\omega * \kappa(x, y)=\int_{X^{2}} \omega\left(z, z^{\prime}\right) \kappa(x, z) \kappa\left(y, z^{\prime}\right) d \mu(z) d \mu\left(z^{\prime}\right) .
$$

Notice the following two important facts about convolutions. First, that it commutes with the coarse coboundary operator: $D(u * \kappa)=(D u) * \kappa$. And second, that

$$
\omega * \kappa-\omega=D u, \text { where } u(x)=\int_{X} \omega(x, z) \kappa(x, z) d \mu(z) .
$$

This implies that $\omega * \kappa$ is a cocycle, and by Jensen's inequality, that $\|u\|_{\phi} \leq\|\omega\|_{\phi, \kappa}$. This also shows that the convolution induces the identity map in cohomology.

Proposition 3.4. Let $X$ and $Y$ be two complete proper metric spaces equipped with Radon measures $\mu_{X}$ and $\mu_{Y}$ satisfying the conditions (3.3) and satisfying the midpoint property. Let $F: X \rightarrow Y$ be a $(\Lambda, \mathrm{c})$-quasi-isometry between them. Then there are kernels $\kappa, \kappa_{Y}$ in $Y$ and $\kappa_{X}$ in $X$ such that the map

$$
F^{*}: \omega \mapsto(\omega * \kappa) \circ F
$$

induces an isomorphism from $L_{\kappa_{Y}}^{\phi} H^{1}(Y)$ to $L_{\kappa_{X}}^{\phi} H^{1}(X)$.

Proof. Choose $R>\max \left\{\Lambda+2 \mathrm{c}, 5 \mathrm{c}_{Y}\right\}$, and consider the kernel $\kappa$ on $Y$ given by (3.4) with $r=R$. Note that for any $z \in Y$, we have

$$
\frac{v_{X}(1)}{V_{Y}(R)} \leq I(z) \leq \frac{V_{X}(\Lambda(R+2 c))}{v_{Y}(R)}, \text { where } I(z)=\int_{X} \kappa(F(x), z) d \mu_{X}(x),
$$

and $v_{X}, V_{X}, v_{Y}$, and $V_{Y}$, denote the functions defined in 3.3 for $X$ and $Y$ respectively.

We let now

$$
R_{X}=2\left(\frac{R-2 \mathrm{c}}{\Lambda}\right)+\Lambda\left(5 \mathrm{c}_{Y}+1+3 \mathrm{c}\right),
$$

and consider the kernel $\kappa_{X}$ on $X$ given by (3.4) with $r=R_{X}$. Finally, define the kernel $\kappa_{Y}$ on $Y$ given by

$$
\kappa_{Y}\left(z, z^{\prime}\right)=\frac{1}{I(z)} \int_{X^{2}} \kappa_{X}(x, y) \kappa(F(x), z) \kappa\left(F(y), z^{\prime}\right) d \mu_{X}(x) d \mu_{X}(y) .
$$

One checks that $\kappa_{Y}$ is bounded (by (3.8)), that its positivity radius is at least $5 c_{Y}+1$, and that $\kappa_{Y}\left(z, z^{\prime}\right)=0$ if $d\left(z, z^{\prime}\right)>2 R+\Lambda R_{X}+\mathrm{c}$.

By construction, and by Jensen's inequality, $\|(\omega * \kappa) \circ F\|_{\phi, \kappa_{X}} \leq\|\omega\|_{\phi, \kappa_{Y}}$ for any cocycle $\omega$ on $Y$. This shows that $F^{*}$ maps $Z_{\phi, \kappa_{Y}}(Y)$ on $Z_{\phi, \kappa_{X}}(X)$. Moreover, by (3.8) and Jensen's inequality again, one checks that $\|(u * \kappa) \circ F\|_{\phi} \lesssim\|u\|_{\phi}$ for any measurable function 
$u: Y \rightarrow \mathbb{R}$. Since $D((u * \kappa) \circ F)=((D u) * \kappa) \circ F$, we see that $F^{*}$ induces a continuous map from $L_{\kappa_{Y}}^{\phi} H^{1}(Y)$ to $L_{\kappa_{X}}^{\phi} H^{1}(X)$. Since quasi-isometries at bounded distance induce the same maps in cohomology, this shows that $F^{*}$ induces an isomorphism.

3.3. Comparision between the De Rham and the simplicial Orlicz cohomologies. In this section we identify the De Rahm cohomology with the coarse cohomology for a simply connected complete Riemannian manifold $M$ with Ricci curvature bounded from below and positive injectivity radius. We denote by $d x$ the volume element of $M$ and notice that $M$ satisfies conditions (3.3). Also, $M$ is quasi-isometric to a geometric simplicial graph $X_{M}$, see $\operatorname{Kan} 85$.

Let $Z_{\phi}^{\infty}(M)$ be the space of closed smooth differential 1-forms in $M$. Equip it with the Luxembourg norm: for $\omega \in Z^{\infty}(M)$,

$$
\|\omega\|_{\phi}=\inf \left\{\alpha>0: \int_{M} \phi\left(\frac{\left|\omega_{x}\right|}{\alpha}\right) d x \leq 1\right\} \in[0,+\infty] .
$$

Here, for $x \in M,\left|\omega_{x}\right|$ is the operator norm of the linear map $\omega_{x}: T_{x} M \rightarrow \mathbb{R}$. Denote $Z_{\phi}^{\infty}(M):=\left\{\omega \in Z^{\infty}(M):\|\omega\|_{\phi}<\infty\right\}$. Its completion $Z_{\phi}(M)$ is the space of measurable $\phi$-integrable 1-forms on $M$ which have zero weak exterior derivative.

The exterior derivative $d: C_{\phi}^{\infty}(M) \rightarrow Z_{\phi}^{\infty}(M)$ is a bounded operator when we consider the norm $\|u\|_{\phi}+\|d u\|_{\phi}$ in the space of smooth functions. Let the operator $d$ to be defined in the completion of $C_{\phi}^{\infty}(M)$; that is, the space of $\phi$-integrable functions on $M$ which have $\phi$-integrable weak derivative. We define the Orlicz-De Rham first cohomology group of $M$ as the quotient normed space $L^{\phi} H^{1}(M):=Z_{\phi}(M) / \operatorname{Im} d$. By integration, we can show that

$$
L^{\phi} H^{1}(M) \simeq\left\{u \in L_{\mathrm{loc}}^{1}(M):\|d u\|_{\phi}<\infty\right\} /\left\{u \in L^{\phi}(M):\|d u\|_{\phi}<\infty\right\} \oplus \mathbb{R} .
$$

The next lemma is a minor generalization of [Pan89a, Lemma 1.12].

Lemma 3.5. For any $0<r<\operatorname{inj}(M)$, there exist a kernel $\kappa_{r}$ on $M$ and a constant $\mathrm{C}_{r}$ such that for any $u \in L_{\text {loc }}^{1}(M)$ with $\|d u\|_{\phi}<\infty$, the following inequality holds

$$
\|D u\|_{\phi, \kappa_{r}} \leq \mathrm{C}_{r}\|d u\|_{\phi} .
$$

The constant $\mathrm{C}_{r}$ depends only on $r$ and $\phi$.

Proof. For a point $x \in M$, let $(v, t) \in T_{x}^{1} M \times(0, r], y=\exp (t v)$, be the polar coordinates with origin $x$. Let $\pi: T^{1} M \rightarrow M$ be the canonical projection. We denote by $\varrho(x, y)$ the volume element in that coordinates, so $\varrho(x, y)^{-1} d y=d t d v$. Notice that $\varrho(x, y)=\varrho(y, x)$ a.e., see [Pan89a, Lemma 1.12]. Let also $\varphi_{s}$ be the geodesic flow.

By the assumptions on $u$, there exists a sequence of smooth functions $u_{n}$ on $M$ which converges to $u$ in $L_{\mathrm{loc}}^{\phi}(M)$ and a.e., and such that $d u_{n}$ converges to $d u$ in $L^{\phi}(M)$. For each $n \in \mathbb{N}, x \in M$ and $v \in T^{1} M$, we have

$$
\left|u_{n}\left(\pi\left(\varphi_{t}(v)\right)\right)-u_{n}(x)\right| \leq \int_{0}^{t}\left|d u_{n}\left(\varphi_{s}(v)\right)\right| d s .
$$

Taking limit as $n \rightarrow \infty, u$ satisfies (3.9) a.e.. Note that by convexity, the function $\phi(t) / t$ is increasing. For $\alpha>0$, Jensen's inequality implies

$$
\phi\left(\frac{u\left(\pi\left(\varphi_{t}(v)\right)\right)-u(x)}{\alpha}\right) \leq \frac{1}{r} \int_{0}^{t} \phi\left(\frac{r\left|d u\left(\varphi_{s}(v)\right)\right|}{\alpha}\right) d s,
$$


Integrating this inequality over $T_{x}^{1} M \times(0, r]$, we get

$$
\int_{B(x, r)} \phi\left(\frac{u(y)-u(x)}{\alpha}\right) \varrho(x, y)^{-1} d y \leq \int_{T_{x}^{1} M} \int_{0}^{r} \phi\left(\frac{r\left|d_{\pi \varphi_{t}(v)} u\right|}{\alpha}\right) d t d v \text {, for a.e. } x \in M \text {. }
$$

Integrating now over $x \in M$, we obtain

$$
\int_{d(x, y) \leq r} \phi\left(\frac{u(y)-u(x)}{\alpha}\right) \varrho(x, y)^{-1} d x d y \leq \operatorname{Vol}\left(S^{n-1}\right) r \int_{M} \phi\left(\frac{r\left|d_{x} u\right|}{\alpha}\right) d x .
$$

Letting $\kappa_{r}(x, y)=\min \left\{1, \varrho(x, y)^{-1}\right\} \mathbb{1}_{\{d(x, y) \leq r\}}$, we obtain $\|D u\|_{\phi, \kappa_{r}} \lesssim\|d u\|_{\phi}$, with a multiplicative constant depending only on $r$ and $\phi$.

In the statement of the next proposition, we denote $\kappa_{y}: M \rightarrow \mathbb{R}$, with $y \in M$, the function $\kappa_{y}(x)=\kappa(x, y)$, where $\kappa$ is a kernel on $M$. The next proposition is a generalization to the general Orlicz case of [Pan89a, Prop. 1.13].

Proposition 3.6. Let $M$ be a simply connected complete Riemannian manifold with Ricci curvature bounded from below and positive injectivity radius. Let $\kappa$ be a smooth kernel on $M$ satisfying in addition that

$$
\mathrm{C}=\|d \kappa\|_{\infty}<\infty \text { and }\left|d_{x} \kappa_{z}\right| \geq \delta>0 \text { if } d(x, z) \leq \varepsilon_{\kappa} .
$$

Then there exists a kernel $\tilde{\kappa}$ on $M$, depending only on $\kappa$, such that the map $u \mapsto u * \kappa$ induces an isomorphism between $L_{\tilde{\kappa}}^{\phi} H^{1}(M)$ and $L^{\phi} H^{1}(M)$. In particular, the Orlicz-De Rham cohomology of $M$ is isomorphic to the simplicial cohomology $\ell^{\phi} H^{1}\left(X_{M}\right)$.

Proof. Consider the kernel $\tilde{\kappa}$ on $M$ given by

$$
\tilde{\kappa}\left(z, z^{\prime}\right):=\frac{1}{I_{z}} \int_{M}\left|d_{x} \kappa_{z}\right| \kappa\left(x, z^{\prime}\right) d x, \text { where } I_{z}=\int_{M}\left|d_{x} \kappa_{z}\right| d x .
$$

Let $\mathrm{a}=\delta v\left(\varepsilon_{\kappa}\right)$, and $\mathrm{b}=\mathrm{C} V(R)$. Notice that $\mathrm{a} \leq I_{z} \leq \mathrm{b}$, and that

$$
\int_{M^{2}}\left|d_{x} \kappa_{z}\right| \kappa\left(x, z^{\prime}\right) d z d z^{\prime}=\int_{M}\left|d_{x} \kappa_{z}\right| d z \in[\mathrm{a}, \mathrm{b}] .
$$

Let $u \in L_{\text {loc }}^{1}(M)$ with $\|D u\|_{\phi, \tilde{\kappa}}<\infty$. Consider the cocycle $\omega=(D u) * \kappa=D(u * \kappa)$. For any $y \in M$, the smooth function $u * \kappa$ satisfies $d(u * \kappa)=d \omega_{y}$, where $\omega_{y}(x)=\omega(x, y)$. Then

$$
\left|d_{x}(u * \kappa)\right| \leq \int_{M^{2}}\left|u(z)-u\left(z^{\prime}\right)\right|\left|d_{x} \kappa_{z}\right| \kappa\left(x, z^{\prime}\right) d z d z^{\prime}, \text { for all } x \in M .
$$

By Jensen's inequality, for any $\alpha>0$, we get

$$
\int_{M} \phi\left(\frac{\left|d_{x}(u * \kappa)\right|}{\alpha}\right) d x \leq \frac{\mathrm{b}}{\mathrm{a}} \int_{M^{2}} \phi\left(\frac{\mathrm{b}\left|u(z)-u\left(z^{\prime}\right)\right|}{\alpha}\right) \tilde{\kappa}\left(z, z^{\prime}\right) d z d z^{\prime} .
$$

That is, $\|d(u * \kappa)\|_{\phi} \lesssim\|D u\|_{\phi, \tilde{\kappa}}$, where the comparison constant depends only on $\kappa, \phi$, and the geometric data of $M$.

Consider now the bounded operator

$$
\left\{u \in L_{\mathrm{loc}}^{1}(M):\|D u\|_{\phi, \tilde{\kappa}}<\infty\right\} \rightarrow\left\{u \in L_{\mathrm{loc}}^{1}(M):\|d u\|_{\phi}<\infty\right\}, u \mapsto u * \kappa
$$

If $u \in L^{\phi}(M)$, then $\|D u\|_{\phi, \tilde{\kappa}}<\infty$, and therefore $\|d(u * \kappa)\|_{\phi} \lesssim\|D u\|_{\phi, \tilde{\kappa}}<\infty$. This implies that $T: L_{\tilde{\kappa}}^{\phi} H^{1}(M) \rightarrow L^{\phi} H^{1}(M),[u] \mapsto[u * \kappa]$ is a well defined bounded operator. 
We show now that this map is an isomorphism. Let $u \in L_{\text {loc }}^{1}(M)$ be such that $\|d u\|_{\phi}<\infty$. By Lemma 3.5, we have $\|D u\|_{\phi, \tilde{\kappa}}<\infty$. Moreover, since

$$
u * \kappa(x)-u(x)=\int_{M}(u(y)-u(x)) \kappa(x, y) d y
$$

by Jensen's inequality we get $\|u * \kappa-u\|_{\phi} \lesssim\|D u\|_{\phi, \tilde{\kappa}}$. Then,

$$
\|u * \kappa-u\|_{\phi}+\|d(u * \kappa-u)\|_{\phi} \lesssim\|D u\|_{\phi, \tilde{\kappa}}+\|d u\|_{\phi}<\infty .
$$

That is, $[u * \kappa]=[u]$, and in particular, $T$ is surjective.

Suppose now that $u \in L_{\text {loc }}^{1}(M),\|D u\|_{\phi, \tilde{\kappa}}<\infty$, and $[u * \kappa]=0$ in $L^{\phi} H^{1}(M)$. Since we also have $\|D u\|_{\phi, \kappa}<\infty$, we can apply (3.10) and conclude that $u * \kappa-u \in L^{\phi}(M)$. Then, $u \in L^{\phi}(M)$ and $[u]=0$ in $L_{\tilde{\kappa}}^{\phi}(M)$. This shows that $T$ is an isomorphism.

REMARK. Note that the proof of the previous proposition also shows that in each cohomology class there is a smooth function: in fact, since $\kappa$ is a smooth kernel the convolution $u * \kappa$ is a smooth function and $[u * \kappa]=[u]$ in $L^{\phi} H^{1}(M)$.

\section{Radial limits and Orlicz-Besov sPaCes}

In this section we prove Theorem 1.2. In the sequel, we assume that $X$ is a uniformly contractible, Gromov hyperbolic, quasi-starlike simplicial complex with bounded geometry. We assume also that $\partial X$ admits a visual metric $\varrho_{w}$ of visual parameter $e$, where $w \in X$ is a base point, which is Ahlfors regular of dimension $Q>0$. Recall that $H$ denotes the $Q$-dimensional Hausdorff measure of $\varrho_{w}$.

REMARK. The contractibility assumption is not a restriction in the hyperbolic case. Indeed, a well known theorem of E. Rips [BH99, Section 3.23], assures that there is always a uniformly contractible Rips complex $\tilde{X}$ of $X$. The inclusion map of $X$ into $\tilde{X}$ is a quasi-isometry, and $\tilde{X}_{0}=X_{0}$. In particular, by Theorem 3.1, the space $\ell^{\phi} H^{1}(\tilde{X})$ does not depend on the particular choice of $\tilde{X}$ up to isomorphism of topological vector spaces.

Since $X$ is contractible, the differential operator $\delta_{0}$ induces an isomorphism

$$
\ell^{\phi} H^{1}(X) \simeq\left\{f: X_{0} \rightarrow \mathbb{R}: \delta_{0} f \in \ell^{\phi}\left(X_{1}\right)\right\} /\left(\ell^{\phi}\left(X_{0}\right)+\mathbb{R}\right),
$$

where $\mathbb{R}$ denotes the constant functions on $X_{0}$. Here, the space on the right is equipped with the topology induced by $\left\|\delta_{0} f\right\|_{\phi}$. In this section we will write $d$ instead of $\delta_{0}$.

4.1. Spherical coordinates and radial shift. Let $\mathcal{R}_{w}$ be the compact space of geodesic rays starting at $w$, and let $\pi_{w}: \mathcal{R}_{w} \rightarrow \partial X$ be the projection $\pi_{w}(\theta)=\lim _{r \rightarrow+\infty} \theta(r)$. Consider $\theta: \partial X \rightarrow \mathcal{R}_{w}, \xi \mapsto \theta_{\xi}$, a measurable section of $\pi_{w}$. For $r \geq 1, \theta_{\xi}[r]$ denotes the edge of $\theta_{\xi}$ at distance $r-1$ from $w$.

We use $|x|$ for the distance $|x-w|$. For $r \in \mathbb{N}$, let $\Sigma_{r}=\{|x|=r\}$, and $A_{r}$ be the set of edges of $X$ with one extremity in the sphere $\Sigma_{r-1}$ and the other one in $\Sigma_{r}$. If $\sigma \in A_{r}$, we denote by $\sigma_{+}$(resp. $\left.\sigma_{-}\right)$the extremity of $\sigma$ in $\Sigma_{r}\left(\right.$ resp. $\left.\Sigma_{r-1}\right)$.

Define the "volume element" by $v:=e^{Q}$, and let $\mu_{v}$ be the discrete measure on $\mathbb{N}$ assigning the mass $v^{r}$ at $r \in \mathbb{N}$. In the product space $\partial X \times \mathbb{N}$, we consider the product measure $H \otimes \mu_{v}$. When a $\phi$-norm is computed in this product space, it will be understood that it is with respect to this measure. By the spherical coordinates on $X$, we mean the map $\partial X \times \mathbb{N} \rightarrow X_{1}$ given by $(\xi, r) \mapsto \theta_{\xi}[r]$.

Consider the radial shift map $S: \partial X \times \mathbb{N} \rightarrow \partial X \times \mathbb{N}$ given by $S(\xi, r)=(\xi, r+1)$. If $G: \partial X \times \mathbb{N} \rightarrow \mathbb{R}$ is any function, we write $S^{*} G$ for the function $G \circ S$. 
Lemma 4.1. Let $\phi$ be a doubling Young function with doubling constant $\mathrm{K}$. The radial shift defines a bounded operator $S^{*}: L^{\phi}(\partial X \times \mathbb{N}) \rightarrow L^{\phi}(\partial X \times \mathbb{N})$ with $\left\|S^{*}\right\| \leq v^{-1 / \mathrm{K}}$.

Proof. By Lemma 2.2, we have $x^{\mathrm{K}} \phi(y) \leq \phi(x y)$ for any $x \in[0,1]$. Let $G \in L^{\phi}(\partial X \times \mathbb{N})$ and take $\alpha>0$. Then

$$
\begin{aligned}
\int_{\partial X} \sum_{r=1}^{\infty} \phi\left(\frac{S^{*} G(\xi, r)}{\alpha}\right) v^{r} d H(\xi) & =\int_{\partial X} \sum_{r=2}^{\infty} \frac{1}{v} \phi\left(\frac{G(\xi, r)}{\alpha}\right) v^{r} d H(\xi) \\
& \leq \int_{\partial X} \sum_{r=1}^{\infty} \phi\left(\frac{G(\xi, r)}{v^{1 / \mathrm{K}} \alpha}\right) v^{r} d H(\xi) .
\end{aligned}
$$

Then, for $\alpha=v^{-1 / \mathrm{K}}\|G\|_{\phi}$, the last integral is equal to one. This proves the lemma.

An immediate consequence of Lemma 4.1 is that the operator

$$
T:=\sum_{k=0}^{\infty}\left(S^{*}\right)^{k}
$$

is bounded. Its norm is bounded by a uniform constant depending only on $\mathrm{K}$ and $v$.

4.2. The radial limit. Let $f: X_{0} \rightarrow \mathbb{R}$ be such that $d f \in \ell^{\phi}\left(X_{1}\right)$. For $\xi \in \partial X$, we set

$$
f_{\infty}(\xi)=\lim _{r \rightarrow \infty} f\left(\theta_{\xi}(r)\right)
$$

if the limit exists. To make the notation simpler, we write $\Delta_{\xi, r}=d f\left(\theta_{\xi}[r]\right), r \in \mathbb{N}$. Define $F: \partial X \times \mathbb{N} \rightarrow \mathbb{R}$ and $D F: \partial X \times \mathbb{N} \rightarrow \mathbb{R}$ to be the functions given by

$$
F(\xi, r)=\sum_{k \geq r} \Delta_{\xi, k} \text { and } D F(\xi, r)=\Delta_{\xi, r} .
$$

Our goal is to show that $F$ is well defined. We first need some notation.

For $x \in X$ and $R>0$, define the shadow

$$
\mho_{w}(x, R):=\left\{\xi \in \partial X: \exists \theta \in \mathcal{R}_{w} \text {, asymptotic to } \xi \text {, with } \theta \cap B_{X}(x, R) \neq \emptyset\right\} .
$$

For $R$ big enough, there is a constant $C_{R} \geq 1$, such that for any $x \in X$, there exists $\xi \in \partial X$ with

$$
B\left(\xi, \mathrm{C}_{R}^{-1} e^{-|x|}\right) \subset \mho_{w}(x, R) \subset B\left(\xi, \mathrm{C}_{R} e^{-|x|}\right) .
$$

See for example [Coo93]. We fix such a big enough $R$ and we write $\mho(x)$ instead of $\mho_{w}(x, R)$. Notice that $H(\mho(x)) \asymp v^{-|x|}$.

Lemma 4.2. Let $f: X_{0} \rightarrow \mathbb{R}$ be any function. Then, $\|D F\|_{\phi} \lesssim\|d f\|_{\phi}$. In particular, if $d f \in \ell^{\phi}\left(X_{1}\right)$, then $D F \in L^{\phi}(\partial X \times \mathbb{N})$.

Proof. We first prove that there exists a constant $C \geq 1$, such that for any function $f: X_{0} \rightarrow \mathbb{R}$ and any $\alpha>0$, we have

$$
\int_{\partial X} \sum_{r=1}^{\infty} \phi\left(\frac{\Delta_{\xi, r}}{\alpha}\right) v^{r} d H(\xi) \leq \mathrm{C} \sum_{\sigma \in X_{1}} \phi\left(\frac{d f(\sigma)}{\alpha}\right) .
$$

For a fixed edge $\sigma \in A_{r}$, the set of points $\xi \in \partial X$ such that $\theta_{\xi}[r]=\sigma$, is contained in the shadow $\mho\left(\sigma_{+}\right)$. Then, there is a uniform constant $\mathrm{C}$ such that $H\left(\xi: \theta_{\xi}[r]=\sigma\right) \leq \mathrm{C} v^{-r}$. 
Applying Fubini's theorem, this implies that

$$
\begin{aligned}
\int_{\partial X} \sum_{r=1}^{\infty} \phi\left(\frac{\Delta_{\xi, r}}{\alpha}\right) v^{r} d H(\xi) & =\sum_{r=1}^{\infty} \sum_{\sigma \in A_{r}} \int_{\left\{\xi: \theta_{\xi}[r]=\sigma\right\}} \phi\left(\frac{d f(\sigma)}{\alpha}\right) v^{r} d H(\xi) \\
& \leq \mathrm{C} \sum_{r=1}^{\infty} \sum_{\sigma \in A_{r}} \phi\left(\frac{d f(\sigma)}{\alpha}\right) \leq \mathrm{C} \sum_{\sigma \in X_{1}} \phi\left(\frac{d f(\sigma)}{\alpha}\right) .
\end{aligned}
$$

When $\|d f\|_{\phi}<\infty$, it suffices to take $\alpha>0$ so that the sum on the right hand side of (4.3) is less than or equal to $\mathrm{C}^{-1}$.

Notice that

$$
F(\xi, r)=\sum_{k=r}^{\infty} \Delta_{\xi, k}=\sum_{k=0}^{\infty} \Delta_{\xi, r+k}=\left(\sum_{k=0}^{\infty}\left(S^{*}\right)^{k} D F\right)(\xi, r),
$$

that is, $F=T(D F)$. As an immediate consequence, an inequality "à la Strichartz" holds (compare with [BP03, Lemma 3.3] and [Str83]): there exists a constant $C:=\|T\|$, depending only on $\phi$ and $v$, such that for any $f: X_{0} \rightarrow \mathbb{R}$ with $d f \in \ell^{\phi}\left(X_{1}\right)$, we have

$$
\|F\|_{\phi} \leq C\|D F\|_{\phi} .
$$

This will be a key point later in the proof of Theorem 1.2 .

We summarize the consequences of inequality (4.4) in the next corollary. For each $r \geq 1$, define the function $f_{r}: \partial X \rightarrow \mathbb{R}$ by setting $f_{r}(\xi)=f\left(\theta_{\xi}(r-1)\right)$. Note that the image of $f_{r}$ is a finite set.

Corollary 4.3 (The radial limit). Let $f: X_{0} \rightarrow \mathbb{R}$ be a function such that $d f \in \ell^{\phi}\left(X_{1}\right)$. Then the radial limit $f_{\infty}(\xi)$ exists $H$-almost everywhere. Moreover:

(1) the function $f_{\infty} \in L^{\phi}(\partial X, H)$,

(2) $f_{r} \rightarrow f_{\infty}$ in $L^{\phi}(\partial X)$ when $r \rightarrow \infty$, and $\left\|f_{\infty}-f(w)\right\|_{\phi} \lesssim\|d f\|_{\phi}$.

Proof. First, note that $F(\cdot, r)=f_{\infty}-f_{r}$. Then, for any $\alpha>0$, we have

$$
\sum_{r=1}^{\infty}\left[\int_{\partial X} \phi\left(\frac{f_{\infty}(\xi)-f_{r}(\xi)}{\alpha}\right) d H(\xi)\right] v^{r}=\int_{\partial X} \sum_{r=1}^{\infty} \phi\left(\frac{F(\xi, r)}{\alpha}\right) v^{r} d H(\xi) .
$$

By letting $\alpha=\|F\|_{\phi}$ and noticing that $f_{1} \equiv f(w)$, we obtain

$$
\left\|f_{\infty}-f(w)\right\|_{\phi} \leq\|F\|_{\phi} \lesssim\|d f\|_{\phi} .
$$

Furthermore, taking $\alpha=1$ in the above equality, we see that

$$
\int_{\partial X} \phi\left(f_{\infty}(\xi)-f_{r}(\xi)\right) d H(\xi) \rightarrow 0, \text { when } r \rightarrow \infty .
$$

Since $\phi$ is doubling, $\left\|f_{\infty}-f_{r}\right\|_{\phi} \rightarrow 0$ when $r \rightarrow \infty$, and the limit is also in $L^{\phi}(\partial X)$.

REMARK. The existence of the radial limit can be proved under the slightly weaker assumption of $\phi$ being of polynomial decay. The cost is that the limit is in $L^{1}(\partial X, H)$. On the other hand, the radial limit does not necessarily exists when $\phi$ decays very fast (like the function $\left.\phi(t)=t^{p} \exp (-1 / t), p \geq 1\right)$. The constraints for a function to be $\phi$-integrable in that case are very weak near infinity. Nevertheless, this kind of functions could be useful in situations were the boundary is not well defined, for instance, in absence of hyperbolicity, or when the space has infinite growth $(v=\infty)$. 
4.3. Proof of Theorem 1.2. Theorem 4.3 ensures the existence of a continuous map

$$
\mathcal{T}:\left\{f: X_{0} \rightarrow \mathbb{R}: d f \in \ell^{\phi}\left(X_{1}\right)\right\} / \mathbb{R} \rightarrow L^{\phi}(\partial X, H) / \mathbb{R}, f \mapsto f_{\infty} .
$$

In this section we determine the image and the kernel of $\mathcal{T}$.

Proposition 4.4 (The kernel of $\mathcal{T}$ ). The kernel of $\mathcal{T}$ is precisely $\left(\ell^{\phi}\left(X_{0}\right) \oplus \mathbb{R}\right) / \mathbb{R}$. In particular, the $\ell^{\phi}$-cohomology is reduced in degree one.

The proof follows from the next lemma which compares the $\phi$-norms of $f$ and $F$.

Lemma 4.5. Let $\phi$ be any Young function, and let $f: X_{0} \rightarrow \mathbb{R}$ be such that $d f \in \ell^{\phi}\left(X_{1}\right)$ and $f_{\infty}=0 H$-almost everywhere. Then, $f \in \ell^{\phi}\left(X_{0}\right)$ if and only if $F \in L^{\phi}(\partial X \times \mathbb{N})$.

Proof of Proposition 4.4. It is clear that if $f \in \ell^{\phi}\left(X_{0}\right)$, then $f_{\infty} \equiv 0$. Suppose that $f_{\infty}=0$ $H$-a.e. and that $d f \in \ell^{\phi}\left(X_{1}\right)$. Then, $F \in L^{\phi}(\partial X \times \mathbb{N})$ by Lemma 4.2 and inequality 4.4. Therefore, $f \in \ell^{\phi}\left(X_{0}\right)$ by Lemma 4.5 .

We will need for the proof of Lemma 4.5, the fact that the shadows $\left\{\mho(x): x \in \Sigma_{r}\right\}$ form a family of coverings of $\partial X$ with the following nice properties:

(1) There is a small constant $\mathrm{c}>0$ such that for each $r \in \mathbb{N}$, the shadow $\mho(x)$, with $x \in \Sigma_{r}$, contains a ball $B(x)$ of radius $\mathrm{c}^{-r}$, and such that for each $r \in \mathbb{N}$, the balls $\left\{B(x): x \in \Sigma_{r}\right\}$ are pairwise disjoint.

(2) There is a constant D such that if $\xi \in \mho(x)$, with $x \in \Sigma_{r}$, then $\left|\theta_{\xi}(r)-x\right| \leq \mathrm{D}$.

(3) For any $\xi \in \partial X$ and $r \in \mathbb{N}$, the number

$$
m_{\xi, r}=\#\left\{x \in \Sigma_{r}: \xi \in \mho(x)\right\} \leq \mathrm{M},
$$

for some uniform constant $\mathrm{M}$.

Proof of Lemma 4.5. Note that since $f_{\infty}=0$ almost everywhere, then $F(\xi, r)=f_{r}(\xi)$ almost everywhere. Denote by $V(\xi, r)$ the ball of radius $\mathrm{D}$ in $X$ centered at $\theta_{\xi}(r)$. By the convexity of $\phi$, for any $\alpha>0$, we have

$$
\phi\left(\frac{f(x)-f_{r}(\xi)}{\mathrm{D} \alpha}\right) \leq \sum_{\sigma \subset V(\xi, r)} \phi\left(\frac{d f(\sigma)}{\alpha}\right) \text { for all } x \in \Sigma_{r}, \xi \in \mho(x), r \in \mathbb{N} .
$$

Define $\tilde{F}: \partial X \times \mathbb{N} \rightarrow \mathbb{R}$ as the average $\tilde{F}(\xi, r)=\frac{1}{m_{\xi, r}} \sum_{x \in \Sigma_{r}} f(x) \mathbb{1}_{\mho(x)}(\xi)$. The convexity of $\phi$ again and (4.6) imply that

$$
\phi\left(\frac{\tilde{F}(\xi, r)-F(\xi, r)}{\mathrm{D} \alpha}\right) \leq \sum_{\sigma \subset V(\xi, r)} \phi\left(\frac{d f(\sigma)}{\alpha}\right), \text { for any } \xi \in \partial X, r \in \mathbb{N} .
$$

Note that if $\xi \in \mho(y)$ for $y \in \Sigma_{r}$, then $V(\xi, r) \subset B(y, 2 \mathrm{D})$. Integrating the last inequality in $\mho(y)$ first, and then summing over $y \in X_{0}$, we obtain

$$
\begin{aligned}
\sum_{r=1}^{\infty} \int_{\partial X} \phi\left(\frac{\tilde{F}(\xi, r)-F(\xi, r)}{\mathrm{D} \alpha}\right) v^{r} d H(\xi) & \leq \sum_{r=1}^{\infty} \sum_{y \in \Sigma_{r}} \int_{\mho(y)} \phi\left(\frac{\tilde{F}(\xi, r)-F(\xi, r)}{\mathrm{D} \alpha}\right) v^{r} d H(\xi) \\
& \lesssim \sum_{r=1}^{\infty} \sum_{y \in \Sigma_{r}} \sum_{\sigma \in B(y, 2 \mathrm{D})} \phi\left(\frac{d f(\sigma)}{\alpha}\right) \\
& \lesssim \sum_{\sigma \in X_{1}} \phi\left(\frac{d f(\sigma)}{\alpha}\right),
\end{aligned}
$$


where the multiplicative constants depend only on the Ahlfors regularity of $H$, the degree of $X$, and $\phi$. Therefore, $\|\tilde{F}-F\|_{\phi} \lesssim\|d f\|_{\phi}<+\infty$. That is, $F \in L^{\phi}(\partial X \times \mathbb{N})$ if, and only if, $\tilde{F}$ is.

We end the proof by comparing the norms of $f$ and $\tilde{F}$. Recall from the definition of the constant $\mathrm{M}$ in 4.5), that we have $m_{\xi, r} \leq \mathrm{M}$. Then, if $x \in \Sigma_{r}$, we have

$$
\begin{aligned}
\phi\left(\frac{f(x)}{\mathrm{M} \alpha}\right) & \lesssim \int_{B(x)} \phi\left(\frac{\tilde{F}(\xi, r)}{\alpha}\right) v^{r} d H(\xi) \\
& \leq \int_{\mho(x)} \phi\left(\frac{\tilde{F}(\xi, r)}{\alpha}\right) v^{r} d H(\xi) \lesssim \sum_{y \in B(x, 2 \mathrm{D})} \phi\left(\frac{f(y)}{\alpha}\right) .
\end{aligned}
$$

Summing over $x \in \Sigma_{r}$ first, and then over $r \in \mathbb{N}$, the same arguments as before show that $f \in \ell^{\phi}\left(X_{0}\right)$ if, and only if, $\tilde{F} \in L^{\phi}(\partial X \times \mathbb{N})$. This finishes the proof.

We now describe the image of $\mathcal{T}$. In the proof of the next proposition, we use the following estimate for the density of the measure $\lambda$ in the space of pairs $\partial^{2} X$ (see the Introduction):

$$
\frac{1}{\rho(\xi, \zeta)^{2 Q}} \asymp \sum_{\sigma \in G_{1}} \frac{\mathbb{1}_{\mho\left(\sigma_{-}\right)}(\xi) \mathbb{1}_{\mho\left(\sigma_{+}\right)}(\zeta)}{H\left(\mho\left(\sigma_{-}\right)\right) H\left(\mho\left(\sigma_{+}\right)\right)}
$$

See [BP03, Lemma 3.5] for the proof.

Proposition 4.6 (The image of $\mathcal{T}$ ). The image of $\mathcal{T}$ is precisely the Orlicz-Besov space $B^{\phi}\left(\partial X, \varrho_{w}\right) / \mathbb{R}$, and $\mathcal{T}$ is continuous in the Orlicz-Besov norm.

Proof. Let $u$ be a measurable function on $\partial X$ with $\langle u\rangle_{\phi}<\infty$. Consider the function $f: X_{0} \rightarrow \mathbb{R}$ given by the averages of $u$ over the shadows:

$$
f(x):=f_{\mho(x)} u(\xi) d H(\xi) .
$$

By Lebesgue's differentiation theorem, the radial limit $f_{\infty}$ exists $H$-almost everywhere and is equal to $u$. We must show that $d f \in \ell^{\phi}\left(X_{1}\right)$. Let $\alpha>0$, by Jensen's inequality

$$
\begin{aligned}
\phi\left(\frac{d f(\sigma)}{\alpha}\right) & =\phi\left(f_{\mho\left(\sigma_{-}\right) \times \mho\left(\sigma_{+}\right)} \frac{u(\xi)-u(\zeta)}{\alpha} d H(\xi) d H(\zeta)\right) \\
& \leq f_{\mho\left(\sigma_{-}\right) \times \mho\left(\sigma_{+}\right)} \phi\left(\frac{u(\xi)-u(\zeta)}{\alpha}\right) d H(\xi) d H(\zeta) .
\end{aligned}
$$

Summing over $\sigma \in X_{1}$, and using the inequalities (4.7), we obtain

$$
\sum_{\sigma \in X_{1}} \phi\left(\frac{d f(\sigma)}{\alpha}\right) \lesssim \int_{\partial^{2} X} \phi\left(\frac{u(\xi)-u(\zeta)}{\alpha}\right) d \lambda(\xi, \zeta) .
$$

This shows that $d f \in \ell^{\phi}\left(X_{1}\right)$ and $\|d f\|_{\phi} \lesssim\langle u\rangle_{\phi}$.

Conversely, let $f: X_{0} \rightarrow \mathbb{R}$ be such that $d f \in \ell^{\phi}\left(X_{1}\right)$. Let $\alpha>0$ and $\sigma \in X_{1}$. Then, by the convexity of $\phi$, we have

$$
\begin{aligned}
f_{\mho\left(\sigma_{-}\right) \times \mho\left(\sigma_{+}\right)} \phi( & \left.\frac{f_{\infty}(\xi)-f_{\infty}(\zeta)}{3 \alpha}\right) d H(\xi) d H(\zeta) \leq \\
& f_{\mho\left(\sigma_{-}\right)} \phi\left(\frac{F\left(\xi,\left|\sigma_{-}\right|\right)}{\alpha}\right) d H(\xi)+f_{\mho\left(\sigma_{+}\right)} \phi\left(\frac{F\left(\zeta,\left|\sigma_{+}\right|\right)}{\alpha}\right) d H(\zeta)+\phi\left(\frac{d f(\sigma)}{\alpha}\right) .
\end{aligned}
$$


In order to bound from above the right hand side of the last inequality, notice that if $\mathrm{M}$ denotes the constant defined in (4.5), then for any $r \in \mathbb{N}$, we have

$$
\sum_{x \in \Sigma_{r}} \mathbb{1}_{\mho(x)} \leq \mathrm{M}\left(1+\sum_{x \in \Sigma_{r}} \mathbb{1}_{B(x)}\right) .
$$

Thus,

$$
\begin{aligned}
\sum_{r=1}^{\infty} \sum_{x \in \Sigma_{r}} \int_{\mho(x)} \phi\left(\frac{F(\xi, r)}{\alpha}\right) v^{r} d H(\xi) & \leq \mathrm{M}\left(1+\sum_{r=1}^{\infty} \sum_{x \in \Sigma_{r}} \int_{B(x)} \phi\left(\frac{F(\xi, r)}{\alpha}\right) v^{r} d H(\xi)\right) \\
& \leq \mathrm{M}\left(1+\sum_{r=1}^{\infty} \int_{\partial X} \phi\left(\frac{F(\xi, r)}{\alpha}\right) v^{r} d H(\xi)\right) .
\end{aligned}
$$

By Lemma 4.2 and inequality (4.4), the know that $\|F\|_{\phi} \lesssim\|d f\|_{\phi}$. Then $\left\langle f_{\infty}\right\rangle_{\phi} \lesssim\|d f\|_{\phi}$. This finishes the proof.

\section{LOCALIZATION}

In this section we prove Theorem 1.3 . We will always assume that $X$ is a uniformly contractible, quasi-starlike, Gromov hyperbolic simplicial complex, with bounded geometry, and that $\phi$ is a doubling Young function.

5.1. Horospherical coordinates and hororadial shift. We first recall the definition and properties of the parabolic visual metrics defined on the punctured boundary $\partial_{\xi} X:=$ $\partial X \backslash\{\xi\}$. For a geodesic ray $r:(-\infty, 0] \rightarrow X$, denote the horofunction (or Busemann function) at $r$ by $b_{r}: X \rightarrow \mathbb{R}$, and recall thar the parabolic Gromov product from $r$ is given by

$$
(x, y)_{r}:=\frac{1}{2}\left(b_{r}(x)+b_{r}(y)-d(x, y)\right) .
$$

Denote by $\xi=r(-\infty) \in \partial X$. This product can be extended to $\partial_{\xi} X$ in the same way as the usual Gromov product, and the function $\varrho_{r}(\zeta, \eta)=e^{-(\zeta, \eta)_{r}}$ is a quasi-metric on $\partial_{\xi} X$. Without loss of generality, we assume that $\varrho_{r}$ is a distance. We call it a parabolic visual metric. It is comparable on compact subsets of $\partial_{\xi} X$ to the usual global visual metric.

This metric is well adapted to the study $\operatorname{Isom}(X, \xi)$, the group of isometries of $X$ which fix the point $\xi$. More precisely, suppose that $F \in \operatorname{Isom}(X, \xi)$, and define the quantity $\Lambda_{r}(F)=e^{-b_{r}(F(w))}$. Then,

$$
\varrho_{r}(F(\zeta), F(\eta)) \asymp \Lambda_{r}(F) \varrho_{r}(\zeta, \eta), \forall \zeta, \eta \in \partial_{\xi} X .
$$

That is, $F$ is a quasi-similarity in the metric $\varrho_{r}$.

We define the shadow from $\xi$ of a ball in $X$ as

$$
\mho_{\xi}(x, R):=\left\{\zeta \in \partial_{\xi} X: \exists \theta=(\xi, \zeta), \theta \cap B(x, R) \neq \emptyset\right\},
$$

where $(\xi, \zeta)$ denotes a geodesic from $\xi$ to $\zeta$. If the radius $R$ is fixed large enough, there is a uniform constant $C \geq 1$ such that, for any $x \in X$, there is a point $\zeta \in \partial_{\xi} X$ with

$$
B\left(\zeta, C^{-1} e^{-b_{r}(x)}\right) \subset \mho_{\xi}(x, R) \subset B\left(\zeta, C e^{-b_{r}(x)}\right) .
$$

From now on, we fix such an $R$ and we write $\mho_{\xi}(x)$ instead of $\mho_{\xi}(x, R)$.

We remark that the $(\partial X, \varrho)$ is $Q$-regular if, and only if, $\left(\partial_{\xi} X, \varrho_{r}\right)$ is $Q$-regular for some (and therefore for any) $\xi \in \partial X$, see [Wil08, Section 6]. We assume $Q$-regularity and 
denote by $H_{r}$ the $Q$-dimensional Hausdorff measure of $\varrho_{r}$. It follows from (5.1), that if $F \in \operatorname{Isom}(X, \xi)$, then $F^{*} H_{r}$ is absolutely continuous with respect to $H_{r}$, and

$$
\frac{d F^{*} H_{r}}{d H_{r}}(\zeta) \asymp \Lambda_{r}(\mathrm{~F})^{Q} .
$$

This implies that the measure

$$
d \lambda_{r}(\zeta, \eta)=\frac{d H_{r} \otimes d H_{r}(\zeta, \eta)}{\varrho_{r}(\zeta, \eta)^{2 Q}},
$$

is almost preserved by $\operatorname{Isom}(X, \xi)$. In particular, the Orlicz-Besov norm of a measurable function $u: \partial_{\xi} X \rightarrow \mathbb{R}$,

$$
\langle u\rangle_{\phi, \xi}:=\inf \left\{\alpha>0: \int_{\partial_{\xi}^{2} X} \phi\left(\frac{u(\zeta)-u(\eta)}{\alpha}\right) d \lambda_{r}(\zeta, \eta) \leq 1\right\},
$$

is almost preserved by such isometries. This fact will be important later in Section 6 .

By a horosphere centred at $\xi$ we mean a level set $H_{c}=\left\{b_{r}(x)=c\right\}$ of the horofunction $b_{r}$. Notice that $H_{0}$ is the horosphere passing through $r(0)$. Since

$$
b_{r}(x) \underset{x \rightarrow \xi}{\rightarrow}-\infty, \text { and } b_{r}(x) \underset{x \rightarrow \zeta}{\rightarrow}+\infty, \forall \zeta \in \partial_{\xi} X,
$$

$H_{c}$ intersects any geodesic from $\xi$ to $\zeta \neq \xi$.

Denote by $\mathcal{G}_{\xi}$ the set of geodesics $\theta: \mathbb{R} \rightarrow X$ with $\theta(-\infty)=\xi$, and $\pi_{\xi}: \mathcal{G}_{\xi} \rightarrow \partial_{\xi} X$ the projection $\pi_{\xi}(\theta)=\theta(+\infty)$. As in Section 4 , we denote by $\theta: \partial_{\xi} X \rightarrow \mathcal{G}_{\xi}$ a measurable section of $\pi_{\xi}$.

Let $\theta$ be a geodesic in $\mathcal{G}_{\xi}$. Since $b_{r}$ is 1-Lipschitz, there is at least one vertex of $X$ in the intersection $\theta \cap\left\{c_{2} \leq b_{r} \leq c_{1}\right\}$ if $c_{1}-c_{2} \geq 1$. We parametrize each geodesic $\theta_{\zeta}$ in such a way that $\theta_{\zeta}(0)$ is a vertex of $X$ in the set $\left\{-1 / 2 \leq b_{r} \leq 1 / 2\right\}$. Notice that there is a uniform constant $C \geq 1$ such that for any $\zeta \in \partial_{\xi} X$,

$$
\left|b_{r}\left(\theta_{\zeta}(s)\right)-s\right| \leq \mathrm{C}+\frac{1}{2} .
$$

For $\zeta \in \partial_{\xi} X$ and $h \in \mathbb{Z}$, we denote by $\theta_{\zeta}[h]:=\sigma$ the edge of $\theta_{\zeta}$ with extremities

$$
\sigma_{-}=\theta_{\zeta}(h-1) \text { and } \sigma_{+}=\theta_{\zeta}(h) \text {. }
$$

As in Section 4, we will also write $v=e^{Q}$, and we define $\mu_{v}$ to be the discrete measure in $\mathbb{Z}$ assigning the mass $v^{h}$ at $h \in \mathbb{Z}$. In the product space $\partial_{\xi} X \times \mathbb{Z}$, we will always consider the product measure $H_{r} \otimes \mu_{v}$. By the horospherical coordinates on $X$ we mean the map $\partial_{\xi} X \times \mathbb{Z} \rightarrow X$ given by $(\zeta, h) \mapsto \theta_{\xi}[h]$.

The hororadial shift $S: \partial_{\xi} X \times \mathbb{Z} \rightarrow \partial_{\xi} X \times \mathbb{Z},(\zeta, h) \mapsto(\zeta, h+1)$, induces a contraction $S^{*}$ on $L^{\phi}\left(\partial_{\xi} X \times \mathbb{Z}\right)$. The same proof as in Lemma 4.1 holds here. Therefore, the operator $T$ defined as in (4.1), is bounded.

5.2. Local $\ell^{\phi}$-cohomology. We first prove that $\ell_{\mathrm{loc}}^{\phi} H^{1}\left(X_{k}, \xi\right)$ is a Fréchet space, we refer to Definition 1.2 for the definition. To this end, we start by showing that there are enough quasi-isometric embeddings on $X$ with uniform constants.

Lemma 5.1. There exist a constant $\mathrm{C} \geq 1$, which depends only on the geometric data of $X$, and a sequence $\left\{Y_{n}\right\}_{n \in \mathbb{N}}$ of hyperbolic geometric complexes, such that

(1) each $Y_{n}$ is a subcomplex of $X$ with $\partial Y_{n} \subset \partial_{\xi} X$,

(2) the inclusion map of $Y_{n}$ into $X$ is a $(1, \mathrm{C})$-quasi-isometric embedding. 
Moreover, the image of any quasi-isometric embedding $\iota \in \mathcal{Y}(X, \xi)$ is contained in $Y_{n}$ for $n$ large enough.

Proof. Denote by $\delta_{\text {hyp }}$ and $\mathrm{C}_{\text {st }}$ the hyperbolicity and quasi-starlike constants of $X$. Fix a geodesic ray $r:(-\infty, 0] \rightarrow X$ with $w=r(0)$ and $\xi=r(-\infty)$. For a compact set $K \subset \partial_{\xi} X$, we define the cone with base $K$ and height $l \in \mathbb{Z} \cup\{-\infty\}$ as

$$
\operatorname{con}(K, l):=\left\{x \in X: b_{r}(x) \geq l \text {, and } \exists \text { a geodesic }(\xi, \zeta) \ni x, \text { with } \zeta \in K\right\} .
$$

By hyperbolicity of $X$, the infinite cone $\operatorname{con}(K,-\infty)$ is $\mathrm{C}_{0}$-quasiconvex, where $\mathrm{C}_{0}$ is a constant depending only on $\delta_{\text {hyp }}$. Since all the geodesics defining the cone are asymptotic to $\xi$, if $l:=l_{K}$ is negative enough, the cone $\operatorname{con}(K, l)$ is $C_{1}$-quasiconvex for a uniform constant $\mathrm{C}_{1}$. Consider the subcomplex $Y_{K}$ of $X$ formed by all the simplices of $X$ which intersect the $\mathrm{C}_{2}$-neighborhood of $\operatorname{con}(K, l)$, where $\mathrm{C}_{2}=\max \left\{\mathrm{C}_{1}, \mathrm{C}_{\mathrm{st}}\right\}$.

We equip $Y_{K}$ with the length metric obtained by restricting the length structure of $X$. From the quasiconvexity of $\operatorname{con}(K, l)$, one checks that the inclusion $\iota: Y_{K} \rightarrow X$ is a $(1, \mathrm{C})$ quasi-isometric embedding, where $\mathrm{C}$ is a constant depending only on $\mathrm{C}_{2}$. In particular, $Y_{K}$ is Gromov hyperbolic, with hyperbolicity constant equal to $3 \mathrm{C}+\delta_{\text {hyp }}$. By construction, $\iota_{\infty}\left(\partial Y_{K}\right)=K$.

Consider the parabolic visual metric $\varrho_{r}$, and take a point $\zeta \in \partial_{\xi} X$. For each $n \in \mathbb{N}$, consider the subcomplex $Y_{n}:=Y_{K_{n}}$ associated to the closed ball $K_{n}=B_{\rho_{r}}[\zeta, n]$ in the above construction. They form an increasing sequence of subcomplexes whose union is the whole $X$. One checks that they verify the desired properties (1) and (2).

It is clear that $\ell_{\mathrm{loc}}^{\phi}\left(X_{k}, \xi\right)$ is a vector space. Moreover, by Lemma 5.1, it is a Fréchet space. In fact, $\left\{\|\cdot\|_{\phi, Y_{n}}: n \in \mathbb{N}\right\}$ is a defining family of seminorms for $\ell_{\text {loc }}^{\phi}\left(X_{k}, \xi\right)$, and a translation invariant metric can be defined as usual by

$$
D_{\phi, \xi}\left(\tau_{1}, \tau_{2}\right)=\sum_{n \in \mathbb{N}} \frac{1}{2^{n}} \max \left\{1,\left\|\tau_{1}-\tau_{2}\right\|_{\phi, Y_{n, l}}\right\} .
$$

Let $\iota: Y \rightarrow X$ be a quasi-isometric embedding in $\mathcal{Y}(X, \xi)$, and denote by $\delta_{k}$ and $\delta_{k, Y}$ the coboundary operators of $X$ and $Y$ respectively. Since $\iota^{*} \circ \delta_{k}=\delta_{k, Y} \circ \iota^{*}$, and since the norm of $\delta_{k, Y}: \ell^{\phi}\left(Y_{k}\right) \rightarrow \ell^{\phi}\left(Y_{k+1}\right)$ is bounded from above by a constant depending only on the geometric data of $Y$, we see that

$$
\delta_{k}: \ell_{\mathrm{loc}}^{\phi}\left(X_{k}, \xi\right) \rightarrow \ell_{\mathrm{loc}}^{\phi}\left(X_{k+1}, \xi\right)
$$

is a Lipschitz continuous operator in the Fréchet distance $D_{\phi, \xi}$.

We now focus on the proof of Theorem 1.3 . First, we observe that

$$
\ell_{\mathrm{loc}}^{\phi} H^{1}(X, \xi) \simeq\left\{f: X_{0} \rightarrow \mathbb{R}: d f \in \ell_{\mathrm{loc}}^{\phi}\left(X_{1}, \xi\right)\right\} /\left(\ell_{\mathrm{loc}}^{\phi}\left(X_{0}, \xi\right) \oplus \mathbb{R}\right) .
$$

The approach follows exactly the same arguments as in Section 4 , but in this case, we work with the horospherical coordinates on $X$.

If $f: X_{0} \rightarrow \mathbb{R}$ is any function and $\zeta \in \partial_{\xi} X$, we set $\Delta_{\zeta, h}=d f\left(\theta_{\zeta}[h]\right), h \in \mathbb{Z}$. Furthermore, let $F: \partial_{\xi} X \times \mathbb{Z} \rightarrow \mathbb{R}$ and $D F: \partial_{\xi} X \times \mathbb{Z} \rightarrow \mathbb{R}$ be the functions defined by

$$
F(\zeta, h)=\sum_{k \geq h} \Delta_{\zeta, k} \text { and } D F(\zeta, h)=\Delta_{\zeta, h}
$$


We denote by $l_{n} \in \mathbb{Z}$ the height of the cone defining $Y_{n}$ in Lemma 5.1. We write $\mathbb{Z}_{n}=$ $\left\{k \in \mathbb{Z}: k \geq l_{n}\right\}$. Then, as in the proof of (4.4), we have

$$
\|F\|_{\phi, K_{n} \times \mathbb{Z}_{n}} \lesssim\|D F\|_{\phi, K_{n} \times \mathbb{Z}_{n}} \text {, for all } n \in \mathbb{N} .
$$

Note that for a fixed edge $\sigma \in X_{1}$ and $h \in \mathbb{Z}$, the set of points $\zeta \in \partial_{\xi} X$ such that $\theta_{\zeta}[h]=\sigma$ is contained in the shadow $\mho_{\xi}\left(\sigma_{+}\right)$. In particular, from (5.4) and (5.2), we have

$$
H_{r}\left(\xi: \theta_{\xi}[h]=\sigma\right) \lesssim v^{-h} .
$$

It follows, exactly as for 4.2 , that

$$
\|D F\|_{\phi, K_{n} \times \mathbb{Z}_{n}} \lesssim\|d f\|_{\phi, Y_{n}}, \text { for all } n \in \mathbb{N} .
$$

Therefore, if $f: X_{0} \rightarrow \mathbb{R}$ is such that $d f \in \ell_{\text {loc }}^{\phi}\left(X_{1}, \xi\right)$, then the hororadial limit $f_{\infty}$ exists almost everywhere, and $f_{\infty} \in L_{\text {loc }}^{\phi}\left(\partial_{\xi} X, H_{r}\right)$. Moreover, if we denote by $f_{h}: \partial_{\xi} X \rightarrow \mathbb{R}$ the function $F(\cdot, h)$, then $f_{h} \rightarrow f_{\infty}$ in $L_{\text {loc }}^{\phi}\left(\partial_{\xi} X, H_{r}\right)$ when $h \rightarrow+\infty$.

Define the trace map from $\xi$ by

$$
\mathcal{T}_{\xi}:\left\{f: X_{0} \rightarrow \mathbb{R}: d f \in \ell_{\mathrm{loc}}^{\phi}\left(X_{1}, \xi\right)\right\} / \mathbb{R} \rightarrow L_{\mathrm{loc}}^{\phi}\left(\partial_{\xi} X, H_{r}\right) / \mathbb{R}, f \mapsto f_{\infty} .
$$

We can determine the kernel and the image of $\mathcal{T}_{\xi}$ as we $\operatorname{did}$ for $\mathcal{T}$.

Proof of Theorem 1.3. We explain how to adapt the proofs of Propositions 4.4 and 4.6 given in Section 4. First, notice that for each subcomplex $Y_{n}$, the intersection $Y_{n} \cap\left\{b_{r} \leq 0\right\}$ has finite diameter. In particular, in order to show that a function $f: X_{0} \rightarrow \mathbb{R}$ is locally $\phi$-integrable, it is enough to bound from above the norm $\|f\|_{\phi, Y_{n} \cap\left\{b_{r} \geq 0\right\}}$ for all $n \in \mathbb{N}$. Since the sequence of coverings

$$
\left\{\mho_{\xi}(x): x \in X_{0} \cap\left\{h \leq b_{r} \leq h+1\right\}\right\}, h \in \mathbb{N},
$$

of $\partial_{\xi} X$, enjoy the same properties (1), (2), and (3), enumerated before the proof of Lemma 4.5 for the coverings $\left\{\mho(x): x \in \Sigma_{r}\right\}_{r \in \mathbb{N}}$, the same proof as in Lemma 4.5 shows that the kernel of $\mathcal{T}_{\xi}$ is precisely $\left(\ell_{\text {loc }}^{\phi}\left(X_{0}\right) \oplus \mathbb{R}\right) / \mathbb{R}$.

An analogous formula to (4.7) holds for the hororadial shadows. More precisely, the density of the measure $\lambda_{r}$ verifies

$$
\frac{1}{\varrho_{r}(\zeta, \eta)^{2 Q}} \asymp \sum_{\sigma \in X_{1}} \frac{\mathbb{1}_{\mho_{r}\left(\sigma_{-}\right)}(\zeta) \mathbb{1}_{\mho_{r}\left(\sigma_{+}\right)}(\eta)}{H_{r}\left(\mho_{r}\left(\sigma_{-}\right)\right) H_{r}\left(\mho_{r}\left(\sigma_{+}\right)\right)}, \text {for } \zeta, \eta \in \partial_{\xi} X \text {. }
$$

The proof is a minor adaptation of (4.7). Therefore, the same arguments as in Proposition 4.6 show that the image of $\mathcal{T}_{\xi}$ is the local Orlicz-Besov space $B_{\text {loc }}^{\phi}\left(\partial_{\xi} X, \varrho_{r}\right) / \mathbb{R}$.

Remark. Consider the continuous local $\ell^{\phi}$-cohomology

$$
A_{\xi}^{\phi}=A_{\mathrm{loc}}^{\phi}\left(\partial_{\xi} X, \varrho_{r}\right):=\left\{u \in B_{\mathrm{loc}}^{\phi}\left(\partial_{\xi} X, \varrho\right): u \text { is continuous }\right\} .
$$

The space $A_{\mathrm{loc}}^{\phi}\left(\partial_{\xi} X, \varrho_{r}\right)$ is a unital commutative Fréchet algebra when equipped with the countable family of multiplicative seminorms

$$
p_{n}(u):=\|u\|_{\infty, K_{n}}+\langle u\rangle_{\phi, K_{n}}, n \in \mathbb{N} .
$$

Its spectrum $\operatorname{Sp}\left(A_{\xi}^{\phi}\right)$ is a Hausdorff hemicompact topological space invariant by Fréchet algebra isomorphisms. We refer to [Gol90] for background on Fréchet algebras. It is homeomorphic to the quotient $\partial_{\xi} X / \sim_{\phi}$, where the local $\ell^{\phi}$-cohomology classes are defined by setting $\zeta \sim_{\phi} \zeta^{\prime} \in \partial_{\xi}$ if, and only if, $u(\zeta)=u\left(\zeta^{\prime}\right)$ for any function $u \in A_{\xi}^{\phi}$. These classes 
must be preserved, in particular, by the boundary homeomorphism of a self quasi-isometry of $X$ which fixes $\xi$.

The next lemma is relevant to Corollary 1.7

Lemma 5.2. Let $\phi$ be a the doubling Young function such that

$$
\sum_{j=0}^{+\infty} \phi\left(2^{-j}\right) 2^{j Q}<\infty
$$

Suppose that $(\partial X, \varrho)$ is $Q$-regular and let $\xi \in \partial X$. Then $A_{\mathrm{loc}}^{\phi}\left(\partial_{\xi} X, \varrho_{r}\right)$ contains all Lipschitz functions.

This is the case for $\phi_{p, \kappa}$, the doubling Young defined in 1.5$)$, whenever $(p, \kappa)>(Q, 1)$.

Proof. Let $u: \partial_{\xi} X \rightarrow \mathbb{R}$ be a L-Lipschitz function and let $K \subset \partial_{\xi} X$ be a compact set. Denote by $\delta=\operatorname{diam} K$, and for $\zeta \in K$ and $j \in \mathbb{N}$, consider the sets $A_{j}(\zeta)$ defined as $\bar{B}\left(\zeta, 2^{-j} \delta\right) \backslash B\left(\zeta, 2^{-(j+1)} \delta\right)$. Then

$$
\begin{aligned}
\sum_{j=0}^{+\infty} \int_{A_{j}(\zeta)} \frac{\phi(u(\eta)-u(\zeta))}{\varrho_{r}(\eta, \zeta)^{2 Q}} d H_{r}(\eta) & \leq \sum_{j=0}^{+\infty} \int_{A_{j}(\zeta)} \frac{\phi\left(\mathrm{L} \varrho_{r}(\eta, \zeta)\right)}{\varrho_{r}(\eta, \zeta)^{2 Q}} d H_{r}(\eta) \\
& \lesssim \sum_{j=0}^{+\infty} \phi\left(2^{-j}\right) 2^{j Q}<\infty .
\end{aligned}
$$

Integrating over $K$ we obtain the result.

\section{Quasi-Isometries of Heintze groups}

In this section we give the proofs of Theorems 1.1, 1.4, 1.5, and Corollaries 1.8 and 1.10 .

6.1. Preliminaries and notation. Let $X_{\alpha}=N \rtimes_{\alpha} \mathbb{R}$ be a purely real Heintze group and $\mathfrak{x}_{\alpha}=T_{(e, 0)} X_{\alpha}$ be its Lie algebra. The left translations are then given by

$$
d_{(e, 0)} L_{(x, t)}=\left(d_{e} L_{x} \circ \operatorname{Exp}(-t \alpha), 1\right),(x, t) \in X_{\alpha} .
$$

We will identify the subgroup $N \times\{0\}$ with $N$. Let $\mathcal{B}=\left\{\partial_{1}, \ldots, \partial_{n}\right\}$ be a basis of $T_{e} N$ on which $\alpha$ assumes its Jordan canonical form, and denote by $\partial_{t}$ the tangent vector at $s=0$ to the curve $s \mapsto(e, s)$.

Let us fix and describe a negatively curved metric on $X_{\alpha}$. Given $\lambda>0$, denote by $\alpha_{\lambda}$ the derivation $\lambda \alpha$. Notice that $X_{\alpha_{\lambda}}$ is always isomorphic to $X_{\alpha}$. By [Hei74, Theorem 2], if $g_{N}$ is a left invariant metric on $N$ for which the symmetric part of $\alpha$ is positive definite and $\lambda$ is large enough, then

$$
g_{(x, t)}=\tau_{\lambda}(t)^{*} g_{N, x} \oplus d t^{2}
$$

defines a metric on $X_{\alpha_{\lambda}}$ of negative sectional curvature bounded from above by -1 . By [Hei74, Theorem 3], if $b_{1}, \ldots, b_{n}$ are well chosen positive numbers, then the symmetric part of $\alpha$ for the left invariant metric on $N$ which makes $\left\{b_{1} \partial_{1}, \ldots, b_{n} \partial_{n}\right\}$ an orthonormal basis, is positive definite. Here the $b_{i}$ depend only on the dimension $n$ and the eigenvalues of $\alpha$. Therefore, $X_{\alpha_{\lambda}}$ is a $\operatorname{CAT}(-1)$ space when equipped with (6.1).

The vertical lines $\gamma_{x}: t \mapsto(x, t)$ are unit-speed geodesics in $X_{\alpha_{\lambda}}$, and define the boundary point denoted $\infty$. The boundary at infinity of $X_{\alpha_{\lambda}}$ is a topological $n$-sphere, which we identify with the one-point compactification $N \cup\{\infty\}$. The orbits of $N$, the sets $N \times\{t\}$, correspond to the horospheres centered at $\infty$. We denote the Riemannian norm and 
distance induced in $N \times\{t\}$ by $\|\cdot\|_{t}$ and $d_{t}$ respectively. The metric on $X_{\alpha_{\lambda}}$ is then given by

$$
\|(v, s)\|_{(x, t)}^{2}=\left\|\operatorname{Exp}\left(t \alpha_{\lambda}\right) \circ d_{x} L_{x^{-1}}(v)\right\|_{0}^{2}+s^{2},(v, s) \in T_{(x, t)} X_{\alpha_{\lambda}} .
$$

The volume element is $d V(x, t)=e^{\lambda \mu t} d V_{N}(x) d t$, where $d V_{N}$ is the volume element of the corresponding left-invariant metric on $N$, and $\mu:=\operatorname{tr}(\alpha)$ is the trace of $\alpha$.

In $N=\partial_{\infty} X_{\alpha_{\lambda}}$, the parabolic visual metric is comparable to the function defined as

$$
\varrho_{\infty}(x, y) \asymp e^{-t} \text {, where } t=\sup \left\{s \in \mathbb{R}: d_{s}\left(\gamma_{x}(s), \gamma_{y}(s)\right) \leq 1\right\}, x, y \in N .
$$

The assertion follows form the fact that both functions are invariant by left translations in $N$, and admit the one-parameter group of contractions $\left\{\tau_{\lambda}(t): t \in \mathbb{R}\right\}$, which contract by the factor $e^{-t}$. We refer to [HP97, Ham89] for more details. This also implies that the parabolic boundary $\left(N, \varrho_{\infty}, d V_{N}\right)$ is Ahlfors regular of dimension $\lambda \mu$.

Recall that $\mu_{1} \leq \cdots \leq \mu_{d}$ are the distinct eigenvalues of $\alpha$, and that $\alpha$ in the generalized eigenspace $V_{i}$ is a sum of $k_{i}$ Jordan blocks of sizes $m_{i j}$, for $j=1, \ldots, k_{i}$. Write the vectors of $\mathcal{B}$ as $\left\{\partial_{k}^{i j},(i, j, k) \in \mathcal{I}\right\}$, where $\mathcal{I}$ is the set of triples

$$
\mathcal{I}:=\left\{(i, j, k): i \in\{1, \ldots, d\}, j \in\left\{1, \ldots, k_{i}\right\}, k \in\left\{1, \ldots, m_{i j}\right\}\right\}
$$

equipped with the lexicographic order.

6.2. The singular value decomposition of $\operatorname{Exp}(t J)$. In this section we prove a lemma about the asymptotic behaviour when $t \rightarrow+\infty$ of the singular value decomposition of the matrix $M=\operatorname{Exp}(t J)$, where $J$ is the standard nilpotent matrix of size $m$ :

$$
J=J(0, m)=\left(\begin{array}{ccccc}
0 & 1 & 0 & \cdots & 0 \\
0 & 0 & 1 & \cdots & 0 \\
\vdots & \vdots & \vdots & \ddots & \vdots \\
0 & 0 & 0 & \cdots & 1 \\
0 & 0 & 0 & \cdots & 0
\end{array}\right) .
$$

Since any two inner products on $\mathbb{R}^{m}$ are equivalent, for our purposes it suffices to consider the standard one $\langle\cdot, \cdot\rangle$, which makes the standard basis $\left\{e_{i}\right\}_{i=1}^{n}$ orthonormal. The next lemma provide us with the key estimates needed to compute the $L^{p, \kappa}$-cohomology of $X_{\alpha}$.

Lemma 6.1. Let $M=\operatorname{Exp}(t J)$ be as above. Let $\left\{v_{1}, \ldots, v_{m}\right\}$ be an orthonormal basis of eigenvectors for $M^{*} M$. Denote $\lambda_{i}$ the eigenvalue associated with $v_{i}$, and suppose they are ordered $\lambda_{1}<\lambda_{2}<\ldots<\lambda_{m}$. Then the following properties are satisfied:

(1) For each $i=1, \ldots, m, \lambda_{i} \lambda_{m-i+1}=1$. In particular, $\lambda_{\frac{m+1}{2}}=1$ if $m$ is odd.

(2) For each $i=1, \ldots, m$,

$$
\sqrt{\lambda_{i}} \sim \frac{(m-i) !}{(i-1) !} t^{2 i-m-1}, \text { when } t \rightarrow+\infty .
$$

(3) For each $i=1, \ldots, m$, the $\lambda_{i}$-eigenspace $\mathbb{R} v_{i}$ of $M^{*} M$ tends to $\mathbb{R} e_{i}$ when $t \rightarrow+\infty$.

Proof. To prove (1) note that $M^{-1}$ is similar to $M$. More precisely, denote by $R$ the reflexion given by $R\left(e_{i}\right)=(-1)^{i} e_{i}$, then $R M R=M^{-1}$. Therefore $M^{-1}$ and $M$ have the same eigenvalues. This proves (1).

We now prove (2). Let $S$ be the reflexion given by $S\left(e_{i}\right)=e_{m-i+1}$. Then $S M S=M^{*}$, and therefore, $M^{*} M=(S M)^{2}$. This implies that $M^{*} M$ and $S M$ have the same eigenvectors, 
and that $\beta_{i}= \pm \sqrt{\lambda_{i}}, i=1, \ldots, m$, are the eigenvalues of $S M$. Also note that $S M$ is symmetric.

Denote by $T_{k}$ the trace of $(S M)^{k}$, and let

$$
p(x)=\operatorname{det}(x I-S M)=x^{m}+c_{1} x^{m-1}+\cdot+c_{m-1} x+c_{m},
$$

be the characteristic polynomial of $S M$. Using the Newton identities

$$
c_{1}=-T_{1} \text { and } T_{k}+c_{1} T_{k-1}+\cdots+c_{k-1} T_{1}+k c_{k}=0, k=2, \ldots, m,
$$

one shows by induction that

$$
\left|c_{k}\right| \sim \frac{(k-1) !(k-2) ! \cdots 1}{(m-k) !(m-k+1) ! \cdots(m-1) !} t^{k(m-k)} \text { when } t \rightarrow+\infty .
$$

On the other hand, by Vieta's identities we have

$$
c_{k}=(-1)^{k} \sum_{1 \leq i_{1}<\cdots<i_{k} \leq m} \beta_{i_{1}} \cdots \beta_{i_{k}} \sim(-1)^{k} \beta_{m-k+1} \cdots \beta_{m-1} \beta_{m},
$$

which together with 6.5 gives (2).

Let us prove (3). Since $S M$ is symmetric, we have that for each $j=1, \ldots, m$, the eigenvector $v_{j}$ is orthogonal to the image of $S M-\beta_{j} I$. Denote by

$$
w_{i}=S M\left(e_{i}\right)=\sum_{k=m-i+1}^{m} \frac{t^{k-m+i-1}}{(k-m+i-1) !} e_{k}, \text { for } i=1, \ldots, m .
$$

Note that since $R M R=M^{-1}$, it is enough to prove the assertion for $j>(m+1) / 2$. Fix such a $j$, so that $\left|\beta_{j}\right| \rightarrow+\infty$ when $t \rightarrow+\infty$. In this case $1<2 j-m \leq m$. The highest degree of the coefficients of $w_{i}$ is $i-1$, given by the coefficient in $e_{m}$. In particular,

$$
u_{i}=\frac{w_{i}-\beta_{j} e_{i}}{\left\|w_{i}-\beta_{j} e_{i}\right\|} \rightarrow \pm e_{i} \text { when } t \rightarrow+\infty
$$

for $i<2 j-m$. Therefore, any limit point of $v_{j}$ is orthogonal to $\operatorname{span}\left(e_{1}, \ldots, e_{2 j-m-1}\right)$. In particular, the limit points of $v_{m}$ are $\pm e_{m}$.

We consider now the case when $j \leq m-1$ and $i \in\{2 j-m, \ldots, j-1\}$. Let $r=m-j-1$, and define recursively

$$
\begin{aligned}
z_{i}^{(0)} & =w_{i} \\
z_{i}^{(s)} & =\frac{d_{i s}}{t} z_{i+1}^{(s-1)}-z_{i}^{(s-1)}, s=1, \ldots, r,
\end{aligned}
$$

where $d_{i s}$ is chosen so that $z_{i}(s)$ has zero coefficient in $e_{m-(s-1)}\left(\right.$ e.g. $\left.d_{i 1}=i\right)$. Then the the coefficient of $z_{i}(r)$ in $e_{k}$ is zero for $k=m-(r-1), \ldots, m$. Moreover, the highest degree of the coefficients of $z_{i}(r)$ is $i-(r+1)$, given by the coefficient in $e_{m-r}$.

The recursion defined above but starting at $w_{i}-\beta_{i} e_{i}$ instead of $w_{i}$ produces a vector $z_{i}$ in the image of $S M-\beta_{i} I$, which in the case $i-r<2 j-m$ verifies

$$
\frac{z_{i}}{\left\|z_{i}\right\|} \rightarrow \pm e_{i} \text {, when } t \rightarrow+\infty \text {. }
$$

This shows that the limit points of $v_{j}$ are orthogonal to $e_{i}$ for $i \leq 2 j-m+r=j-1$. This finishes the proof of (3). 
Let us apply the previous lemma in our setting. Consider the two inner products on $T_{e} N$, $\langle\cdot, \cdot\rangle$ and $\langle\cdot, \cdot\rangle_{0}$, who make $\left\{\partial_{k}\right\}$ and $\left\{b_{k} \partial_{k}\right\}$ orthonormal basis respectively. Note that $\operatorname{Exp}\left(t \alpha_{\lambda}\right)$ has the diagonal block form

$$
\bigoplus_{i=1}^{d} \bigoplus_{j=1}^{k_{i}} e^{t \mu_{i}} \operatorname{Exp}\left(\lambda t J\left(0, m_{i j}\right)\right)
$$

If $v$ is any vector in $V_{i j}=\operatorname{Span}\left(\partial_{k}^{i j}, k=1, \ldots, m_{i j}\right)$, then

$$
\left\|\operatorname{Exp}\left(t \alpha_{\lambda}\right)(v)\right\|_{0} \asymp e^{\lambda t \mu_{i}} \| \operatorname{Exp}\left(\lambda t J\left(0, m_{i j}\right)(v) \| .\right.
$$

Let $\left\{v_{1}^{i j}(t), \ldots, v_{m_{i j}}^{i j}(t)\right\}$ be an orthonormal basis (for $\left.\langle\cdot, \cdot\rangle\right)$ of $V_{i j}$ formed by singular eigenvectors of $\operatorname{Exp}\left(\lambda t J\left(0, m_{i j}\right)\right)$, and denote by $\lambda_{1}^{i j}(t)<\cdots<\lambda_{m_{i j}}^{i j}(t)$ its singular values. By item (2) of Lemma 6.1, we have

$$
\sqrt{\lambda_{k}^{i j}(t)} \sim \mathrm{c}_{k}^{i j} t^{2 k-m_{i j}-1}, \text { when } t \rightarrow+\infty,
$$

where $c_{k}^{i j}=\lambda^{2 k-m_{i j}-1}\left(m_{i j}-k\right) ! /(k-1)$ !. In particular,

$$
\left\|\operatorname{Exp}\left(t \alpha_{\lambda}\right)\left(v_{k}^{i j}(t)\right)\right\|_{0} \asymp t^{2 k-m_{i j}-1} e^{\lambda t \mu_{i}} \text {, when } t \rightarrow+\infty \text {. }
$$

Moreover, by item (3) of Lemma 6.1, we can chose $v_{k}^{i j}(t)$ so that

$$
\left\|v_{k}^{i j}(t)-\partial_{k}^{i j}\right\|_{0} \rightarrow 0, \text { when } t \rightarrow+\infty
$$

In other words, the singular eigenvectors converge to the standard basis vectors of $V_{i j}$.

6.3. The degree one $L^{p, \kappa}$-cohomology of $X_{\alpha}$ : main computations. Consider $u$ a real smooth function on $X_{\alpha_{\lambda}}$. For each $t \in \mathbb{R}$, denote by $u_{t}: N \rightarrow \mathbb{R}$ the function $u_{t}(x)=u(x, t)$, and let

whenever this limit exists.

$$
u_{\infty}(x):=\lim _{t \rightarrow+\infty} u_{t}(x)
$$

Let $E \subset N$ be a measurable set and $t_{0} \in \mathbb{R}$. As we proved in Section 5.1, the hororadial shift

$$
w \in L^{p, \kappa}\left(E \times\left[t_{0},+\infty\right)\right) \mapsto S_{k}(w)(x, t)=\int_{k}^{k+1} w(x, t+s) d s,
$$

is a contraction in the $\phi_{p, \kappa}$-norm, and its norm is bounded from above by $e^{-k \lambda \mu / \mathrm{K}}$, where $\mathrm{K}$ is the doubling constant of $\phi_{p, \kappa}$. The proof in this case follows from Jensen's inequality. Therefore, the operator

$$
T(w)(x, t)=\sum_{k=0}^{\infty} S_{k}(w)(x, s)=\int_{0}^{+\infty} w(x, t+s) d s,
$$

is bounded in the $\phi_{p, \kappa}$-norm.

Suppose that $d u \in L^{p, \kappa}\left(E \times\left[t_{0},+\infty\right)\right)$. Under mild assumptions on $E$, for instance if $E$ is $\lambda \mu$-Ahlfors regular, the functions $u_{t}$ converge a.e. and in $L_{\text {loc }}^{p, \kappa}\left(E, d V_{N}\right)$ to $u_{\infty}$. In particular, $u_{\infty} \in L_{\mathrm{loc}}^{1}\left(E, d V_{N}\right)$ and $d u_{\infty}$ is well defined in the sense of distributions. Moreover, the operator $T$ evaluated at the function $\partial_{t} u$ gives

$$
T\left(\partial_{t} u\right)(x, t)=u_{\infty}(x)-u_{t}(x) .
$$

Thus, we can write $u_{\infty}=v+u$, where $v \in L^{p, \kappa}\left(E \times\left[t_{0},+\infty\right)\right)$. 
For $(i, j, k) \in \mathcal{I}$, we denote by $X_{k}^{i j}$ the left invariant vector field of $N$ generated by the basis vector $\partial_{k}^{i j}$, and by $Y_{k}^{i j}(t)$ the left invariant vector field of $N$ generated by $v_{k}^{i j}(t)$. Recall from the previous section that $v_{k}^{i j}(t) \in V_{i j}$ is a singular eigenvector of $\operatorname{Exp}\left(t \alpha_{\lambda}\right)$, and $v_{k}^{i j}(t) \rightarrow \partial_{k}^{i j}$ when $t \rightarrow+\infty$. When the pair $(i, j)$ is clear from the context, we simply write $X_{k}, \partial_{k}, Y_{k}(t), v_{k}(t)$, and $m$ respectively. Recall that for $i \in\{1, \ldots, d\}$, the $i$-critical exponent is given by $p_{i}=\mu / \mu_{i}$.

Lemma 6.2. Let $(p, \kappa) \in I$, and let $E$ denotes either $N$ or $N \backslash\{\xi\}$ for some point $\xi$. Consider $u: X_{\alpha_{\lambda}} \rightarrow \mathbb{R}$ a smooth function such that $d u \in L^{p, \kappa}(K \times[t,+\infty))$ for any compact $K \subset E$ and $t \in \mathbb{R}$. Then, $X_{k}^{i j}\left(u_{\infty}\right)=0$ whenever

$$
(p, \kappa) \leq\left(p_{i}, 1+p_{i}\left(m_{i j}+1-2 k\right)\right) .
$$

Proof. Let $u: X_{\alpha_{\lambda}} \rightarrow \mathbb{R}$ be as in the statement. For each $t \geq 0$, consider the function $Y_{k}(t)\left(u_{t}\right): E \rightarrow \mathbb{R}$. We first show that $Y_{k}(t)\left(u_{t}\right) \rightarrow X_{k}\left(u_{\infty}\right)$ in the sense of distributions.

Let $\zeta: E \rightarrow \mathbb{R}$ be a smooth function with compact support $K$. We first observe that

$$
\left\langle\left(Y_{k}(t)-X_{k}\right)\left(u_{t}\right), \zeta\right\rangle=-\int_{E} u_{t}(x) d_{e}\left(\zeta \circ L_{x}\right)\left(v_{k}(t)-\partial_{k}\right) d V_{N}(x) .
$$

Moreover, since $e \in \operatorname{supp}\left(\zeta \circ L_{x}\right)=L_{x}^{-1}(K)$ if, and only if, $x \in K$, we have

$$
\left|\left\langle\left(Y_{k}(t)-X_{k}\right)\left(u_{t}\right), \zeta\right\rangle\right| \leq\left\|u_{t}\right\|_{1, K} \max _{x \in K}\left|d_{e}\left(\zeta \circ L_{x}\right)\right|_{0}\left\|v_{k}(t)-\partial_{k}\right\|_{0}
$$

which tends to zero when $t \rightarrow+\infty$ by item (3) of Lemma 6.1.

Finally, we obtain

$$
\left|\left\langle Y_{k}(t)\left(u_{t}\right), \zeta\right\rangle-\left\langle X_{k}\left(u_{\infty}\right), \zeta\right\rangle\right| \leq\left|\left\langle Y_{k}(t)\left(u_{t}\right)-X_{k}\left(u_{t}\right), \zeta\right\rangle\right|+\left|\left\langle X_{k}\left(u_{t}\right)-X_{k}\left(u_{\infty}\right), \zeta\right\rangle\right|,
$$

which tends to zero since $X_{k}\left(u_{t}\right) \rightarrow X_{k}\left(u_{\infty}\right)$.

We now show that $X_{k}\left(u_{\infty}\right)=0$. By the definition of $v_{k}(t)$ and 6.6), we have

$$
\tau_{k}(t):=\left\|Y_{k}(t)(x)\right\|_{t}=\left\|\operatorname{Exp}(t \alpha)\left(v_{k}(t)\right)\right\|_{0} \asymp t^{2 k-m_{i j}-1} e^{\lambda t \mu_{i}} .
$$

Also

$$
\left|Y_{k}(t)\left(u_{t}\right)(x)\right| \leq \tau_{k}(t)\left|d_{(x, t)} u\right|
$$

Let $c>0$ be a constant to be determined later. Consider the set

$$
E_{t}=\left\{x \in E:\left|Y_{k}(t)\left(u_{t}\right)(x)\right| \geq e^{-c t}\right\} .
$$

Then, for $x \in E_{t}$ we have

$$
\frac{\tau_{k}(t)^{-p}\left|Y_{k}(t)\left(u_{t}\right)(x)\right|^{p}}{\log ^{\kappa}\left(e+\tau_{k}(t) e^{c t}\right)} \leq \phi_{p, \kappa}\left(\frac{\left|Y_{k}(t)\left(u_{t}\right)(x)\right|}{\tau_{k}(t)}\right) \leq \phi_{p, \kappa}\left(\left|d_{(x, t)} u\right|\right) .
$$

Moreover, there exists a constant $\mathrm{C}>0$, and $t_{0}>0$, such that

$$
\frac{1}{\mathrm{C}} \frac{e^{-p \lambda \mu_{i} t}\left|Y_{k}(t)\left(u_{t}\right)(x)\right|^{p}}{t^{\kappa+p\left(2 k-m_{i j}-1\right)}} \leq \frac{\tau_{k}(t)^{-p}\left|Y_{k}(t)\left(u_{t}\right)(x)\right|^{p}}{\log ^{\kappa}\left(e+\tau_{k}(t) e^{\mathrm{c} t}\right)}, \text { for } t \geq t_{0} .
$$

Let $q=\kappa+p\left(2 k-m_{i j}-1\right)$, then for any compact $K \subset E$, we have

$$
A:=\int_{t_{0}}^{+\infty} \int_{E_{t} \cap K} \frac{\left|Y_{k}(t)\left(u_{t}\right)(x)\right|^{p} e^{\lambda\left(\mu-p \mu_{i}\right) t}}{t^{q}} d V_{N}(x) d t \leq \mathrm{C} \int_{K \times\left[t_{0},+\infty\right)} \phi_{p, \kappa}(|d u|) d V<\infty .
$$


This implies

$$
\int_{t_{0}}^{+\infty} \int_{K} \frac{\left|Y_{k}(t)\left(u_{t}\right)(x)\right|^{p} e^{\lambda\left(\mu-p \mu_{i}\right) t}}{t^{q}} d V_{N}(x) d t \leq A+V_{N}(K) \int_{t_{0}}^{+\infty} \frac{e^{\left(-p c+\lambda\left(\mu-p \mu_{i}\right)\right) t}}{t^{q}} d t .
$$

Let $\mathrm{c}>-\lambda p^{-1}\left(\mu-p \mu_{i}\right)$. Therefore, whenever $(p, \kappa)$ satisfies $(6.7)$, there is a subsequence $t_{l} \rightarrow+\infty$ such that

$$
\left\|Y_{k}\left(t_{l}\right)\left(u_{t_{l}}\right)\right\|_{p, K} \rightarrow 0, \text { when } l \rightarrow+\infty .
$$

This shows that $X_{k}\left(u_{\infty}\right)=0$.

We denote by $\left\{\theta_{k}^{i j}\right\}$ the basis of $V_{i j}^{*}$, dual to the standard left invariant basis $\left\{X_{k}^{i j}\right\}$. For each $t \in \mathbb{R}$, we have

$$
\left|\theta_{k}^{i j}\right|_{t}=\left\|\operatorname{Exp}\left(-t \alpha_{\lambda}^{*}\right)\left(\partial_{k}^{i j}\right)\right\|_{0} \asymp\left[\sum_{r=k}^{m_{i j}} \frac{t^{2(r-k)}}{(r-k) !^{2}}\right]^{1 / 2} e^{-\lambda \mu_{i} t} \asymp t^{m_{i j}-k} e^{-\lambda \mu_{i} t} .
$$

In the following lemma, we will use the functions $\mathrm{i}$ and $\mathrm{m}$ that assign to a subset $\mathcal{I}_{0}$ of $\mathcal{I}$ the integers

$$
\begin{aligned}
\mathrm{i}=\mathrm{i}\left(\mathcal{I}_{0}\right) & :=\min \left\{r:(r, s, l) \notin \mathcal{I}_{0}\right\}, \\
\mathrm{m}=\mathrm{m}\left(\mathcal{I}_{0}\right) & :=\max \left\{m_{\mathrm{is}}-l:(\mathrm{i}, s, l) \notin \mathcal{I}_{0}\right\} .
\end{aligned}
$$

Given a smooth function $u_{\infty}: N \rightarrow \mathbb{R}$, by its hororadial extension we mean the function independent of $t, u: X_{\alpha_{\lambda}} \rightarrow \mathbb{R}$, defined by $u(x, t)=u_{\infty}(x)$.

Lemma 6.3. Let $p \geq 1$ and $\kappa \geq 0$. Fix $\mathcal{I}_{0}$ a subset of $\mathcal{I}$ and denote by $\mathrm{i}=\mathrm{i}\left(\mathcal{I}_{0}\right)$ and $\mathrm{m}=\mathrm{m}\left(\mathcal{I}_{0}\right)$. Consider $u_{\infty}: N \rightarrow \mathbb{R}$ a smooth function such that

$$
X_{l}^{r s}\left(u_{\infty}\right)=0 \text { for all }(r, s, l) \in \mathcal{I}_{0} .
$$

Let $u: X_{\alpha_{\lambda}} \rightarrow \mathbb{R}$ be its hororadial extension. Then $d u \in L^{p, \kappa}\left(K \times\left[t_{0},+\infty\right)\right)$ for any compact $K \subset N$ and $t_{0} \in \mathbb{R}$, whenever

$$
(p, \kappa)>\left(p_{\mathrm{i}}, 1+p_{\mathrm{i}} \mathrm{m}\right) .
$$

Moreover, in both cases, if $u_{\infty}$ is not identically zero and the compact $K \subset N$ has positive measure, then $u \notin L^{p, \kappa}(K \times[0,+\infty))$.

Proof. Let $K \subset N$ be a compact set. Since $u$ does not depend on $t$, it is clear that $u \notin L^{p, \kappa}(K \times[0,+\infty))$ when $K$ has positive measure, unless $u_{\infty}$ is identically zero.

If $\mathcal{I}_{0}=\mathcal{I}$, there is nothing to prove since $u$ is constant. So suppose that $\mathcal{I}_{0}$ is a proper subset of $\mathcal{I}$. Let $C$ be an upper bound in $K$ for $\left|X_{l}^{r s}\left(u_{\infty}\right)\right|$, for any $(r, s, l) \in \mathcal{I}$. Then, by (6.8), there is $t_{1}>0$ such that

$$
\left|d_{(x, t)} u\right| \leq \mathrm{C} \sum_{(r, s, l) \notin \mathcal{I}_{0}}\left|\theta_{l}^{r s}\right|_{t} \lesssim \sum_{(r, s, l) \notin \mathcal{I}_{0}} t^{m_{r s}-l} e^{-\lambda \mu_{r} t} \lesssim t^{\mathrm{m}} e^{-\lambda \mu_{\mathrm{i}} t},
$$

for $x \in K$, and $t \geq t_{1}$. Therefore,

$$
\int_{t_{1}}^{+\infty} \int_{K} \phi_{p, \kappa}\left(\left|d_{(x, t)} u\right|\right) e^{\lambda \mu t} d V_{N}(x) d t \lesssim V_{N}(K) \int_{t_{1}}^{+\infty} \frac{e^{\lambda\left(\mu-p \mu_{\mathrm{i}}\right) t}}{t^{\kappa-p m}} d t .
$$

The conclusion follows from this inequality. 
We will apply the previous lemma later to the following subsets of indices. Let $\mathcal{K}_{0}=\emptyset$, and for each $i \in\{1, \ldots, d\}$, let

$$
\mathcal{K}_{i}=\{(r, j, k): r \leq i\}, \mathcal{H}_{i}=\mathcal{K}_{i-1} \cup\left\{(i, j, 1): m_{i j}=m_{i}\right\} .
$$

Then, for $0 \leq i<d$ we have

$$
\mathrm{i}\left(\mathcal{K}_{i}\right)=i+1, \text { and } \mathrm{m}\left(\mathcal{K}_{i}\right)=m_{i+1}-1 .
$$

Also, for any $i$, we have

$$
\mathrm{i}\left(\mathcal{H}_{i}\right)=i \text {, and } \mathrm{m}\left(\mathcal{H}_{i}\right)=m_{i}-2,
$$

if $m_{i} \geq 2$. Notice that if $m_{i}=1$, then $\mathcal{K}_{i}=\mathcal{H}_{i}$.

For $\xi \in N, r>0$ and $t \in \mathbb{R}$, we denote by $Y(r, t)$ the complement in $X_{\alpha_{\lambda}}$ of the set $B(\xi, r) \times(t,+\infty)$, where $B(\xi, r)$ is the open ball of radius $r$ centered at $\xi$ in $N$.

The proof of the next lemma is inspired by [Pan07, Lemma 20].

Lemma 6.4. Let $\xi \in N,(p, \kappa) \in I$, and let $u: X_{\alpha_{\lambda}} \rightarrow \mathbb{R}$ be a smooth function such that $d u \in L^{p, \kappa}(Y(r, t))$, for all $r>0$ and $t \in \mathbb{R}$. Suppose that there is a left-invariant vector field $X$ of $N$, generated by an eigenvector $X_{e}$ of $\alpha$, such that $X\left(u_{\infty}\right)=0$. If $Z$ is a left-invariant vector field of $N$ such that $[X, Z]=0$, then $Z\left(u_{\infty}\right)=0$.

Proof. We denote by $\tilde{X}$ and $\tilde{Z}$ the left-invariant vector fields of $X_{\alpha_{\lambda}}$ generated by $\left(X_{e}, 0\right)$ and $\left(Z_{e}, 0\right)$ respectively. Notice that they coincide with $(X, 0)$ and $(Z, 0)$ in $N$. Let $\tilde{u}_{\infty}$ be the hororadial extension of $u_{\infty}$. Since $X_{e}$ is an eigenvector of $\alpha$, we have $\tilde{X}\left(\tilde{u}_{\infty}\right)=0$. Moreover, $\tilde{X}$ and $\tilde{Z}$ also commute.

First, notice that since the exponential map of $N$ is a diffeomorphism, the one-parameter subgroup $\left\{\exp \left(s\left(X_{e}, 0\right)\right): s \in \mathbb{R}\right\}$ is closed in $X_{\alpha_{\lambda}}$. This implies that the flow of $\tilde{X}$,

$$
\varphi_{s}(x, t)=(x, t) \cdot \exp \left(s\left(X_{e}, 0\right)\right),
$$

satisfies the following property: for any compact subset $K \subset X_{\alpha_{\lambda}}$, there exists $l_{K} \geq 0$ such that if $\left|s-s^{\prime}\right| \geq l_{k}$, then $\varphi_{s}(K) \cap \varphi_{s^{\prime}}(K)=\emptyset$.

Moreover, since $N$ is nilpotent and the coefficient of $\left[\tilde{X}, \partial_{t}\right]$ in $\partial_{t}$ is zero, we see that $\operatorname{tr}\left(\operatorname{ad}_{\tilde{X}}\right)=0$. In particular,

$$
\varphi_{s}^{*}(d V)=e^{-\operatorname{tr}(\operatorname{ad} \tilde{X})} d V=d V,
$$

i.e. the flow $\varphi_{s}$ preserves the measure $d V$.

Suppose that $w: X_{\alpha_{\lambda}} \rightarrow \mathbb{R}$ is a function in $L^{p, \kappa}(Y(r, t))$ for all $r$ and $t$. We claim that for each compact set $K \subset X_{\alpha_{\lambda}}$, we have

$$
\left\|w \circ \varphi_{s}\right\|_{1, K} \rightarrow 0, \text { when } s \rightarrow+\infty .
$$

Indeed, fix such a compact set $K$, and choose $r$ and $t$ so that $\varphi_{s}(K) \subset Y(r, t)$ for all $s \in \mathbb{R}_{+}$. Given any sequence $s_{l} \rightarrow+\infty$, we can extract a subsequence $s_{l^{\prime}} \rightarrow+\infty$ such that the images $\left\{\varphi_{s_{l^{\prime}}}(K): l^{\prime} \in \mathbb{N}\right\}$ are pairwise disjoint. Therefore,

$$
\sum_{l^{\prime} \in \mathbb{N}} \int_{K} \phi_{p, \kappa}\left(w \circ \varphi_{s_{l^{\prime}}}\right) d V=\sum_{l^{\prime} \in \mathbb{N}} \int_{\varphi_{s_{l^{\prime}}}(K)} \phi_{p, \kappa}(w) d V \leq \int_{Y(r, t)} \phi_{p, \kappa}(w) d V<\infty .
$$

This implies that $\left\|w \circ \varphi_{s_{l^{\prime}}}\right\|_{p, \kappa, K} \rightarrow 0$ when $l^{\prime} \rightarrow+\infty$. Since $L^{p, \kappa}(K) \subset L^{1}(K)$ and the inclusion is norm continuous, the claim follows. 
Write $\tilde{u}_{\infty}=w+u$, where $w \in L^{p, \kappa}(Y(r, t))$, for all $r$ and $t$. From the claim above, we have that $w \circ \phi_{s} \rightarrow 0$, when $s \rightarrow+\infty$, in the sense of distributions. Also, since $d u \in L^{p, \kappa}(Y(r, t))$ for all $r$ and $t$, and $\tilde{Z}$ has constant norm, the same is true for the function $\tilde{Z}(u)$

Again by the claim above, we have $\tilde{Z}(u) \circ \varphi_{s} \rightarrow 0$, when $s \rightarrow+\infty$, in the sense of distributions. But since $\tilde{Z}$ commutes with $\tilde{X}$, we have

$$
\tilde{Z}(u) \circ \varphi_{s}=\tilde{Z}\left(u \circ \varphi_{s}\right) \text {, for all } s \in \mathbb{R} .
$$

Therefore,

$$
\tilde{Z}\left(\tilde{u}_{\infty}\right)=\tilde{Z}\left(\tilde{u}_{\infty} \circ \varphi_{s}\right)=\tilde{Z}\left(w \circ \phi_{s}\right)+\tilde{Z}\left(u \circ \varphi_{s}\right) \rightarrow 0,
$$

in the sense of distributions when $s \rightarrow+\infty$. That is, $\tilde{Z}\left(\tilde{u}_{\infty}\right)=0$.

Let $\zeta: N \backslash\left\{x_{0}\right\} \rightarrow \mathbb{R}$ and $\eta: \mathbb{R} \rightarrow \mathbb{R}$ be smooth functions with compact support. Let $\zeta \times \eta(x, t)=\zeta(x) \eta(t)$. Notice that $\tilde{Z}(x, t)=\left(Z_{t}(x), 0\right)$ where $Z_{t}$ is a left-invariant vector field in $N$. Since $\eta$ does not depend on $x \in N$, we have $Z_{t}(\eta)=0$. Then

$$
0=\left\langle\tilde{Z}\left(\tilde{u}_{\infty}\right), \zeta \times \eta\right\rangle=-\int_{\mathbb{R}}\left\langle u_{\infty}, Z_{t}(\zeta)\right\rangle \eta(t) e^{\lambda \mu t} d t .
$$

Since $\zeta$ and $\eta$ where arbitrary, this shows that $Z\left(u_{\infty}\right)=0$.

Corollary 6.5. Let $\xi \in N,(p, \kappa) \in I$, and let $u: X_{\alpha_{\lambda}} \rightarrow \mathbb{R}$ be a smooth function satisfying the hypotheses of Lemma 6.4. Then $u_{\infty}: N \backslash\{\xi\} \rightarrow \mathbb{R}$ is constant a.e.

Proof. Let $Z \neq 0$ be a left-invariant vector field in the center of $\mathfrak{n}$, which exists because $N$ is nilpotent. By Lemma 6.4, $Z\left(u_{\infty}\right)=0$, that is $u_{\infty} \circ \varphi_{s}=u_{\infty}$ a.e., where $\varphi_{s}(x)=$ $R_{\exp (s Z)}(x)=L_{\exp (s Z)}(x)$. Using again the fact that the action $s \mapsto \varphi_{s}$ is proper, we conclude that the Orlicz-Besov norm of $u_{\infty}$ is zero on any compact set of $N \backslash\{\xi\}$. This proves the lemma.

6.4. Proofs of main results. We now apply the results obtained in the previous section to compute the local and global cohomology of $X_{\alpha}$.

Recall that for each $i \in\{1, \ldots, d\}$, we have defined $m_{i}=\max \left\{m_{i j}: 1 \leq j \leq k_{i}\right\}$, and the subspaces $W_{0}=\{0\}, W_{i}=V_{1} \oplus \cdots \oplus V_{i}$, and

$$
\mathfrak{k}_{i}=\operatorname{LieSpan}\left(W_{i}\right), V_{i}^{0}=\operatorname{Span}\left(\partial_{1}^{i j}: m_{i j}=m_{i}\right), \mathfrak{h}_{i}=\operatorname{LieSpan}\left(W_{i-1} \oplus V_{i}^{0}\right) .
$$

We denote also by $K_{i}$ and $H_{i}$ the closed Lie subgroups of $N$ whose Lie algebra are $\mathfrak{k}_{i}$ and $\mathfrak{h}_{i}$ respectively. Note that the set of left cosets $N / K_{i}$ are $N / H_{i}$ are a smooth manifold, and the canonical projections are smooth maps.

Proof of Theorem 1.4. First, suppose that $p \in\left(p_{i+1}, p_{i}\right)$ and $\kappa \geq 0$. Let $u: X_{\alpha} \rightarrow \mathbb{R}$ be a smooth function with $d u \in L^{p, \kappa}\left(K \times\left[t_{0},+\infty\right)\right)$, for any compact set $K \subset N$ and $t_{0} \in \mathbb{R}$. If $i=d$, there is nothing to prove, so suppose $i<d$. Consider the subset $\mathcal{K}_{i}$ defined in 6.9). By Lemma 6.2 $X_{k}^{r j}\left(u_{\infty}\right)=0$ for all $(r, j, k) \in \mathcal{K}_{i}$, and thus $X\left(u_{\infty}\right)=0$ for any left-invariant field $X \in \mathfrak{k}_{i}$. If we suppose $u_{\infty}$ continuous, this implies that $u_{\infty}$ is constant on any left coset of $K_{i}$, and can therefore be regarded as a continuous function on $N / K_{i}$.

Note that $\mathrm{i}\left(\mathcal{K}_{i}\right)=i+1$ and $p>p_{i+1}$. By Lemma 6.3, any smooth function $u_{\infty}$ on $N / H_{i}$ can be identified with the hororadial limit of a smooth function $u: X_{\alpha} \rightarrow \mathbb{R}$ with $d u \in L^{p, \kappa}\left(K \times\left[t_{0},+\infty\right)\right)$, for any compact $K \subset N$ and $t_{0} \in \mathbb{R}$. This shows (1).

Let us prove (2). Suppose that $p=p_{i}$ and $m_{i}=1$. Let $\kappa \geq 0$. By point (1), if $u: X_{\alpha} \rightarrow \mathbb{R}$ is a smooth function with $d u \in L^{p, \kappa}\left(K \times\left[t_{0},+\infty\right)\right)$, for any compact set $K \subset N$ and $t_{0} \in \mathbb{R}$, then $X\left(u_{\infty}\right)=0$ for any $X \in \mathfrak{k}_{i-1}$. 
If $\kappa \leq 1$, because $m_{i}=1$, Lemma 6.2 implies that $X\left(u_{\infty}\right)=0$ for any $X \in \mathfrak{k}_{i}$. If $i=d$, there is nothing more to prove. If not, since $p>p_{i+1}$, the same argument as in (1) concludes the proof of the first part of (2).

If $\kappa>1$, because $\mathrm{i}\left(\mathcal{K}_{i-1}\right)=i$ and $\mathrm{m}\left(\mathcal{K}_{i-1}\right)=m_{i}-1=0$, Lemma 6.3 implies that any smooth function $u_{\infty}$ on $N / K_{i-1}$ can be identified with the hororadial limit of a smooth function $u: X_{\alpha} \rightarrow \mathbb{R}$ with $d u \in L^{p, \kappa}\left(K \times\left[t_{0},+\infty\right)\right)$, for any compact $K \subset N$ and $t_{0} \in \mathbb{R}$. This completes the proof of (2).

Suppose now that $p=p_{i}$ and $1+p_{i}\left(m_{i}-2\right)<\kappa \leq 1+p_{i}\left(m_{i}-1\right)$, with $m_{i} \geq 2$. Consider the subset $\mathcal{H}_{i}$ defined in (6.9). Let $u: X_{\alpha} \rightarrow \mathbb{R}$ be a smooth function with $d u \in L^{p, \kappa}\left(K \times\left[t_{0},+\infty\right)\right)$, for any compact set $K \subset N$ and $t_{0} \in \mathbb{R}$. By Lemma 6.2, $X_{k}^{r j}\left(u_{\infty}\right)=0$ for all $(r, j, k) \in \mathcal{H}_{i}$. Thus $X\left(u_{\infty}\right)=0$ for any left-invariant field $X \in \mathfrak{h}_{i}$. If we suppose $u_{\infty}$ continuous, this implies that $u_{\infty}$ is constant on any left coset of $H_{i}$, and can be regarded as a function on $N / H_{i}$.

Since $\mathrm{i}\left(\mathcal{H}_{i}\right)=i, \mathrm{~m}\left(\mathcal{H}_{i}\right)=m_{i}-2$, and $\kappa>1+p_{i}\left(m_{i}-2\right)$, by Lemma 6.3, any smooth function $u_{\infty}$ on $N / H_{i}$ can be identified with the hororadial limit of a smooth function $u: X_{\alpha} \rightarrow \mathbb{R}$ with $d u \in L^{p, \kappa}\left(K \times\left[t_{0},+\infty\right)\right)$, for any compact $K \subset N$ and $t_{0} \in \mathbb{R}$. This shows (3).

Proof of Theorem 1.5. Let $[u] \in L^{p, \kappa} H^{1}\left(X_{\alpha}, \xi\right)$ be a local cohomology class represented by a smooth function $u: X_{\alpha} \rightarrow \mathbb{R}$. By Lemma 6.2, if

$$
(p, \kappa) \leq\left(p_{1}, 1+p_{1}\left(m_{1}-1\right)\right),
$$

then $X_{1}^{1 j}\left(u_{\infty}\right)=0$, for any $j$ with $m_{1 j}=m_{1}$. Since $X_{1}^{1 j}$ is a left-invariant vector field of $N$ generated by an eigenvector of $\alpha$, we can apply Corollary 6.5 to conclude that $u_{\infty}$ is constant a.e.. Then $[u]=0$.

Suppose that $(p, \kappa)>\left(p_{1}, 1+p_{1}\left(m_{1}-1\right)\right)$. Consider $u_{\infty}$ any smooth function with compact support in $N$. Take $\eta: \mathbb{R} \rightarrow \mathbb{R}$ a smooth function such that $\eta(t)=0$ for $t<0$ and $\eta(t)=1$ for $t \geq 1$. Set $u: X_{\alpha} \rightarrow \mathbb{R}$ as $u(x, t)=\eta(t) u_{\infty}(x)$. Then the hororadial limit of $u$ is $u_{\infty}$, and the same computations as in Lemma 6.3 show that $d u \in L^{p, \kappa}\left(X_{\alpha}\right)$. Since $[u] \neq 0$, this finishes the proof.

Proof of Theorem 1.1. Suppose $X_{\alpha}$ is not of Carnot type. Then, either $m_{1}=1$, so $\mathfrak{h}_{1}$ and $\mathfrak{k}_{1}$ coincide precisely with the proper subalgebra of $\mathfrak{n}$ spanned by the $\mu_{1}$-eigenvectors of $\alpha$; or $m_{1} \geq 2$ and $\mathfrak{h}_{1}$ is a proper Lie subalgebra of $\mathfrak{n}$.

In the first case, we necessarily have $d \geq 2$, and $i(\alpha)=\min \left\{i: K_{i}=N\right\} \geq 2$. In both cases, by Theorems 1.4 and 1.5 , we have

$$
p_{\neq 0}\left(X_{\alpha}, \infty, I\right) \leq\left(p_{i(\alpha)}, 1+p_{i(\alpha)}\left(m_{i(\alpha)}-1\right)^{+}\right)<\left(p_{1}, m_{1}-1\right)=p_{\neq 0}\left(X_{\alpha}, \xi, I\right),
$$

for any $\xi \in N$. This shows that $\infty$ is fixed by the boundary homeomorphism of any quasi-isometry of $X_{\alpha}$, and that it preserves the left cosets of $H_{1}$.

6.5. Proof of Corollary 1.8. Let $N$ be of nilpotency class $\ell$, and let $F \in \operatorname{QIsom}\left(X_{\alpha}\right)$. We must prove that the boundary extension of $F$ is a bi-Lipschitz homeomorphism.

The derivation $\alpha$ induces a Carnot structure on the subgroup $H_{1}$, and therefore, the restriction of the snow-flaked parabolic visual metric $\varrho_{\infty}^{1 / \mu_{1}}$ is bi-Lipschitz equivalent to the Carnto-Carathéodory distance of $H_{1}$, which is, in particular, a geodesic distance. Since $F$ preserves the left cosets of $H_{1}$, by [LX14, Theorem 1.1], it is enough to prove: 
(1) Any left coset is accumulated by parallel lefts cosets: for any $x \in N$, there exists a sequence $x_{j} \rightarrow x, x_{j} H_{1} \neq x H_{2}$, such that

$$
\operatorname{dist}\left(x_{j} H_{1}, x H_{1}\right)=\operatorname{dist}\left(x_{j}^{\prime}, x H_{1}\right)=\operatorname{dist}\left(x_{j} H_{1}, x^{\prime}\right),
$$

for all $x_{j}^{\prime} \in x_{j} H$ and $x^{\prime} \in x H$.

(2) Left cosets diverge sub-linearly: for any $x, y \in N$, there exists a sequence $x_{j} \in x H_{1}$ such

$$
\frac{\operatorname{dist}\left(x_{j}, y H_{1}\right)}{\varrho_{\infty}\left(x_{j}, x\right)} \rightarrow 0, \text { when } j \rightarrow+\infty .
$$

To prove (1), let $z_{j}$ be a sequence in the normalizer of $H_{1}$, not in $H_{1}$, and such that $z_{j} \rightarrow e$. Then $x_{j}=x z_{j}$ satisfies (1) because $\varrho_{\infty}$ is left-invariant.

Let us prove (2). Let $x, y \in N$ and write $x^{-1} y x=\exp (Y)$, with $Y \in \mathfrak{n}$. For $t \geq 0$, let $x_{t}=x \exp (t X)$ with $X \in \mathfrak{h}_{1}$ a $\mu_{1}$-eigenvector of $\alpha$. Then $\varrho_{\infty}\left(x_{t}, y x_{t}\right)=\varrho_{\infty}\left(e, x_{t}^{-1} y x_{t}\right)$. By the Baker-Campbell-Hausdorff formula,

$$
x_{t}^{-1} y x_{t}=\exp \left(Y^{\prime}+p(Y, t X)\right)
$$

where $Y^{\prime}$ does not depend on $t$ and $p$ is a polynomial of degree $\ell-1$. Without loss of generality, we can suppose that $\varrho_{\infty}\left(e, x_{t}^{-1} y x_{t}\right) \rightarrow+\infty$ when $t \rightarrow+\infty$.

By definition, $\varrho_{\infty}(e, z)=e^{s}$ if, and only if, $d_{0}(e, \tau(s)(z))=1$, where we recall that $d_{0}$ is the left-invariant Riemannian distance on $N$. Notice that $d_{0}(e, \exp (Z)) \leq\|Z\|_{0}$ for any $Z \in \mathfrak{n}$. Moreover, there is a constant $\delta>0$ such that

$$
\delta \leq d_{0}(e, \exp (Z)) \leq 1 \text { whenever }\|Z\|_{0}=1 .
$$

First, observe that

$$
\operatorname{Exp}(-s \alpha)\left(\left[\partial_{k}^{i j}, \partial_{l}^{r s}\right]\right)=e^{-\left(\mu_{i}+\mu_{r}\right) s} \sum_{h=1, h^{\prime}=1}^{k, l} \frac{(-s)^{m_{i j}+m_{r s}-k-l}}{\left(m_{i j}-k\right) !\left(m_{r s}-l\right) !}\left[\partial_{k}^{i j}, \partial_{l}^{r s}\right]
$$

This implies, by letting $Z_{s}:=\operatorname{Exp}(-s \alpha)(p(Y, t X))$, that

$$
\left\|Z_{s}\right\|_{0} \leq \sum_{h=1}^{\ell-1} t^{h} e^{-(h+1) \mu_{1} s} q_{h}(s) \lesssim \sum_{h=1}^{\ell-1} t^{h} e^{-(h+1 / 2) \mu_{1} s},
$$

where $q_{h}(s)$ are polynomials in $s$, and the last inequality holds for $s$ large enough. Choosing $s=s_{t}$ so that $d_{0}\left(e, \exp \left(t Z_{s}\right)\right)=1$, we obtain from the last inequality

$$
s_{t} \leq \frac{\ell}{(\ell+1 / 2) \mu_{1}} \log t+O(1) .
$$

Let $X_{s}=\operatorname{Exp}(-s \alpha)(X)$. A similar, but simpler, computation shows that for

$$
s^{*}=s_{t}^{*}:=\frac{1}{\mu_{1}} \log t+O(1),
$$

we have $\delta \leq d_{0}\left(e, \exp \left(t X_{s^{*}}\right)\right) \leq 1$. Putting everything together, we obtain

$$
\frac{d\left(x_{t}, y x_{t}\right)}{d\left(x_{t}, x\right)} \lesssim e^{s_{t}-s_{t}^{*}} \rightarrow 0
$$

when $t \rightarrow+\infty$. This concludes the proof of (2). 
6.6. The abelian case: proof of Corollary 1.10. Suppose that $N \simeq \mathbb{R}^{n}$ is abelian, and let $X_{\alpha}$ and $X_{\beta}$ be two quasi-isometric purely real Heintze groups. Notice that being of Carnot type in this case is equivalent to the derivation being a scalar multiple of the identity. Therefore, we can assume that neither $X_{\alpha}$ nor $X_{\beta}$ is of Carnot type. We will use the same notations as before, but we add a superscript $\alpha$ or $\beta$ to indicate to which derivation it corresponds.

Write $\alpha$ and $\beta$ in their Jordan form

$$
\alpha=\bigoplus_{i=1}^{d^{\alpha}} \bigoplus_{j=1}^{k_{i}^{\alpha}} J\left(\mu_{i}^{\alpha}, m_{i j}^{\alpha}\right), \beta=\bigoplus_{i=1}^{d^{\beta}} \bigoplus_{j=1}^{k_{i}^{\beta}} J\left(\mu_{i}^{\beta}, m_{i j}^{\beta}\right) .
$$

Consider the functions $s^{\alpha}$ and $s^{\beta}$ defined on the index set $\{(p, \kappa): p \geq 1, \kappa \geq 0\}$ which give the topological dimension of the spectrum of the local cohomology with respect to $\infty$.

Since $N$ is abelian, we have $\mathfrak{k}_{i}^{\alpha}=W_{i}^{\alpha}$ and $\mathfrak{k}_{i}^{\beta}=W_{i}^{\beta}$. By Theorem 1.4, we know that

$$
s^{\alpha}(p, \kappa)=n-\sum_{r=1}^{i} \operatorname{dim} V_{r}^{\alpha} \text { and } s^{\beta}(p, \kappa)=n-\sum_{r=1}^{i} \operatorname{dim} V_{r}^{\beta},
$$

for $p \in\left(p_{i+1}^{\alpha}, p_{i}^{\alpha}\right)$ and $p \in\left(p_{i+1}^{\beta}, p_{i}^{\beta}\right)$ respectively. Note that at each critical exponent $s_{\alpha}$ and $s_{\beta}$ jump and change their values. Since $s_{\alpha}=s_{\beta}$, we conclude that $d^{\alpha}=d^{\beta}=d$, and $\operatorname{dim} V_{i}^{\alpha}=\operatorname{dim} V_{i}^{\beta}$ for all $i=1, \ldots, d$. In particular,

$$
\frac{\mu_{i}^{\alpha}}{\mu_{i}^{\beta}}=\frac{\operatorname{tr}(\alpha)}{\operatorname{tr}(\beta)}=\lambda>0, i=1, \ldots, d .
$$

On the other hand,

$$
\operatorname{dim} V_{i}^{\alpha}=\sum_{j=1}^{k_{i}^{\alpha}} m_{i j}^{\alpha}, \text { and } \operatorname{dim} V_{i}^{\beta}=\sum_{j=1}^{k_{i}^{\beta}} m_{i j}^{\beta} .
$$

Moreover, by Theorem 1.4 we already know that for each $i \in\{1, \ldots, d\}$,

$$
m_{i}^{\alpha}=\max \left\{m_{i j}^{\alpha}: j=1, \ldots, k_{i}^{\alpha}\right\}=m_{i}^{\beta}=\max \left\{m_{i j}^{\beta}: j=1, \ldots, k_{i}^{\beta}\right\}=m_{i} .
$$

In particular, if $m_{i}=1$ for all $i$ (that is, $\alpha$ and $\beta$ are diagonalizable), then $k_{i}^{\alpha}=k_{i}^{\beta}$ also, and the proof is completed in this case. We will proceed by induction on $d$ and the size of the biggest Jordan block of the first eigenvalue.

Suppose that for $i=2, \ldots, d$ we have $k_{i}^{\alpha}=k_{i}^{\beta}=k_{i}$ and $m_{i j}^{\alpha}=m_{i j}^{\beta}$ for $j=1, \ldots, k_{i}$. If $d=1$ the assumption is empty. We will show that the same is true for $i=1$. This is of course the case if $m_{1}=1$. So assume that $m_{1} \geq 2$.

Let $F: X_{\alpha} \rightarrow X_{\beta}$ be a quasi-isometry and denote also by $F$ its boundary extension. By Theorem 1.4. $F$ induces a homeomorphism $F_{1}: \mathbb{R}^{n} / H_{1}^{\alpha} \rightarrow \mathbb{R}^{n} / H_{1}^{\beta}$. Let $\pi_{\alpha}$ and $\pi_{\beta}$ be the canonical projections onto $\mathbb{R}^{n} / H_{1}^{\alpha}$ and $\mathbb{R}^{n} / H_{1}^{\beta}$ respectively. The linear actions verify

$$
\pi_{\alpha} \circ \operatorname{Exp}(t \alpha)=\operatorname{Exp}\left(t \alpha_{1}\right) \circ \pi_{\alpha} \text { and } \pi_{\alpha} \circ \operatorname{Exp}(t \beta)=\operatorname{Exp}\left(t \beta_{1}\right) \circ \pi_{\beta},
$$

where now the biggest Jordan block of the first eigenvalue is $m_{1}-1$ (notice that $k_{1}^{\alpha_{1}}=k_{1}^{\alpha}$ and $k_{1}^{\beta_{1}}=k_{1}^{\beta}$ ). Let $\varrho_{\alpha}$ and $\varrho_{\beta}$ be the parabolic visual metrics defined before. The key point to apply the induction argument is to notice that the left cosets of $H_{1}^{\alpha}$ and $H_{1}^{\beta}$ are equidistant for $\varrho_{\alpha}$ and $\varrho_{\beta}$ respectively. Let $\tilde{\varrho}_{\alpha}$ and $\varrho_{\beta}$ be the quotient distances. On checks, 
see [Tys01, Lemma 15.9], that $F_{1}$ is a quasi-symmetry in these metrics. Since they are left invariant and the linear actions of $\alpha_{1}$ and $\beta_{1}$ are dilations with dilation expansion $e^{t}$, they are bi-Lipschitz equivalent to the respective parabolic visual metrics $\varrho_{\alpha_{1}}$ and $\varrho_{\beta_{1}}$. This shows that $\left(\mathbb{R}^{n} / H_{1}^{\alpha}, \varrho_{\alpha_{1}}\right)$ is quasi-symmetric to $\left(\mathbb{R}^{n} / H_{1}^{\beta}, \varrho_{\beta_{1}}\right)$, and induction applies.

Note that we have shown, in particular, the case when $d=1$. So suppose that $d \geq 2$ and that the theorem is proved for $d-1$. Considering the quotients by $K_{1}^{\alpha}$ and $K_{1}^{\beta}$ and applying similar arguments as above, we show by induction that for $i=2, \ldots, d$ we have $k_{i}^{\alpha}=k_{i}^{\beta}=k_{i}$ and $m_{i j}^{\alpha}=m_{i j}^{\beta}$ for $j=1, \ldots, k_{i}$. Then we can apply the previous assertion and conclude that $k_{1}^{\alpha}=k_{1}^{\beta}=k_{1}$ and $m_{1 j}^{\alpha}=m_{1 j}^{\beta}$ for all $j=1, \ldots, k_{1}$. This ends the proof.

\section{REFERENCES}

[BH99] Martin R. Bridson and André Haefliger. Metric spaces of non-positive curvature, volume 319 of Grundlehren der Mathematischen Wissenschaften [Fundamental Principles of Mathematical Sciences]. Springer-Verlag, Berlin, 1999.

[BK12] M. Bourdon and B. Kleiner. Some applications of $\ell_{p}$-cohomology to boundaries of Gromov hyperbolic spaces. ArXiv e-prints, March 2012.

[BK13] Marc Bourdon and Bruce Kleiner. Combinatorial modulus, the combinatorial Loewner property, and Coxeter groups. Groups Geom. Dyn., 7(1):39-107, 2013.

[Bou07] Marc Bourdon. Une caractérisation algébrique des homéomorphismes quasi-Möbius. Ann. Acad. Sci. Fenn. Math., 32(1):235-250, 2007.

[BP03] Marc Bourdon and Hervé Pajot. Cohomologie $l_{p}$ et espaces de Besov. J. Reine Angew. Math., 558:85-108, 2003

[Coo93] Michel Coornaert. Mesures de Patterson-Sullivan sur le bord d'un espace hyperbolique au sens de Gromov. Pacific J. Math., 159(2):241-270, 1993.

[Cor12] Y. Cornulier. On the quasi-isometric classification of focal hyperbolic groups. ArXiv e-prints, December 2012.

[CT11] Yves Cornulier and Romain Tessera. Contracting automorphisms and $L^{p}$-cohomology in degree one. Ark. Mat., 49(2):295-324, 2011.

[DP11] Tullia Dymarz and Irine Peng. Bilipschitz maps of boundaries of certain negatively curved homogeneous spaces. Geom. Dedicata, 152:129-145, 2011.

[Dym10] Tullia Dymarz. Large scale geometry of certain solvable groups. Geom. Funct. Anal., 19(6):16501687, 2010.

[FM00] Benson Farb and Lee Mosher. On the asymptotic geometry of abelian-by-cyclic groups. Acta Math., 184(2):145-202, 2000 .

[GdlH90] É. Ghys and P. de la Harpe, editors. Sur les groupes hyperboliques d'après Mikhael Gromov, volume 83 of Progress in Mathematics. Birkhäuser Boston, Inc., Boston, MA, 1990. Papers from the Swiss Seminar on Hyperbolic Groups held in Bern, 1988.

[Gol90] Helmut Goldmann. Uniform Fréchet algebras, volume 162 of North-Holland Mathematics Studies. North-Holland Publishing Co., Amsterdam, 1990.

[Gro93] M. Gromov. Asymptotic invariants of infinite groups. In Geometric group theory, Vol. 2 (Sussex, 1991), volume 182 of London Math. Soc. Lecture Note Ser., pages 1-295. Cambridge Univ. Press, Cambridge, 1993.

[Ham87] Ursula Hamenstädt. Zur Theorie von Carnot-Carathéodory Metriken und ihren Anwendungen. Bonner Mathematische Schriften [Bonn Mathematical Publications], 180. Universität Bonn, Mathematisches Institut, Bonn, 1987. Dissertation, Rheinische Friedrich-Wilhelms-Universität Bonn, Bonn, 1986.

[Ham89] Ursula Hamenstädt. A new description of the Bowen-Margulis measure. Ergodic Theory Dynam. Systems, 9(3):455-464, 1989.

[Hei74] Ernst Heintze. On homogeneous manifolds of negative curvature. Math. Ann., 211:23-34, 1974.

[Hei01] Juha Heinonen. Lectures on analysis on metric spaces. Universitext. Springer-Verlag, New York, 2001.

[HP97] Sa'ar Hersonsky and Frédéric Paulin. On the rigidity of discrete isometry groups of negatively curved spaces. Comment. Math. Helv., 72(3):349-388, 1997. 
[HP11] P. Haïssinsky and K. M. Pilgrim. Minimal Ahlfors regular conformal dimension of coarse conformal dynamics on the sphere. ArXiv e-prints, March 2011.

[Kan85] Masahiko Kanai. Rough isometries, and combinatorial approximations of geometries of noncompact Riemannian manifolds. J. Math. Soc. Japan, 37(3):391-413, 1985.

[Kop13] Y. Kopylov. Amenability of Closed Subgroups and Orlicz Spaces. ArXiv e-prints, May 2013.

[KP13] Y. Kopylov and R. Panenko. $\Phi$-Harmonic Functions on Discrete Groups and First $\ell^{\Phi}$-Cohomology. ArXiv e-prints, November 2013.

[LX14] E. LeDonne and X. Xie. Rigidity of fiber-preserving quasisymmetric maps. Preprint, 2014.

[MT10] John M. Mackay and Jeremy T. Tyson. Conformal dimension, volume 54 of University Lecture Series. American Mathematical Society, Providence, RI, 2010. Theory and application.

[Pan89a] Pierre Pansu. Cohomologie $L^{p}$ des variétés à courbure négative, cas du degré 1. Rend. Sem. Mat. Univ. Politec. Torino, (Special Issue):95-120 (1990), 1989. Conference on Partial Differential Equations and Geometry (Torino, 1988).

[Pan89b] Pierre Pansu. Dimension conforme et sphère à l'infini des variétés à courbure négative. Ann. Acad. Sci. Fenn. Ser. A I Math., 14(2):177-212, 1989.

[Pan89c] Pierre Pansu. Métriques de Carnot-Carathéodory et quasiisométries des espaces symétriques de rang un. Ann. of Math. (2), 129(1):1-60, 1989.

[Pan02] Pierre Pansu. $L^{p}$-cohomology and pinching. In Rigidity in dynamics and geometry (Cambridge, 2000), pages 379-389. Springer, Berlin, 2002.

[Pan07] Pierre Pansu. Cohomologie $L^{p}$ en degré 1 des espaces homogènes. Potential Anal., 27(2):151-165, 2007.

[Pan08] Pierre Pansu. Cohomologie $L^{p}$ et pincement. Comment. Math. Helv., 83(2):327-357, 2008.

[Pen11] Irine Peng. Large scale geometry of nilpotent-by-cyclic groups. Geom. Funct. Anal., 21(4):9511000, 2011.

[RR91] M. M. Rao and Z. D. Ren. Theory of Orlicz spaces, volume 146 of Monographs and Textbooks in Pure and Applied Mathematics. Marcel Dekker, Inc., New York, 1991.

[Shc14] V. Shchur. On the quantitative quasi-isometry problem: transport of Poincare inequalities and different types of quasi-isometric distortion growth. ArXiv e-prints, January 2014.

[Str83] Robert S. Strichartz. Analysis of the Laplacian on the complete Riemannian manifold. J. Funct. Anal., 52(1):48-79, 1983.

[SX12] Nageswari Shanmugalingam and Xiangdong Xie. A rigidity property of some negatively curved solvable Lie groups. Comment. Math. Helv., 87(4):805-823, 2012.

[Tri83] Hans Triebel. Theory of function spaces, volume 78 of Monographs in Mathematics. Birkhäuser Verlag, Basel, 1983.

[Tys01] Jeremy T. Tyson. Metric and geometric quasiconformality in Ahlfors regular Loewner spaces. Conform. Geom. Dyn., 5:21-73 (electronic), 2001.

[Wil08] K. Wildrick. Quasisymmetric parametrizations of two-dimensional metric planes. Proc. Lond. Math. Soc. (3), 97(3):783-812, 2008.

[Xie12] Xiangdong Xie. Quasisymmetric maps on the boundary of a negatively curved solvable Lie group. Math. Ann., 353(3):727-746, 2012.

[Xie13] X. Xie. Quasiisometries of negatively curved homogeneous manifolds associated with Heisenberg groups. ArXiv e-prints, August 2013.

[Xie14a] Xiangdong Xie. Large scale geometry of negatively curved $\mathbb{R}^{n} \rtimes \mathbb{R}$. Geom. Topol., 18(2):831-872, 2014.

[Xie14b] Xiangdong Xie. Rigidity of quasiisometries of hmn associated with non-diagonalizable derivation of the heisenberg algebra. Preprint, 2014.

E-mail address: matias@math.u-psud.fr

Laboratoire de Mathématiques d'Orsay 